\title{
MAXWELL QUASI-VARIATIONAL INEQUALITIES IN SUPERCONDUCTIVITY
}

\author{
IRWIN YOUSEPT*
}

\begin{abstract}
This paper is devoted to the mathematical modeling and analysis of a hyperbolic Maxwell quasi-variational inequality (QVI) for the Bean-Kim superconductivity model with temperature and magnetic field dependence in the critical current. Our analysis relies on local (resp. global) boundedness and local (resp. global) Lipschitz continuity assumptions on the critical current with respect to the temperature (resp. magnetic field). Emerging from the Euler time discretization, we analyze the corresponding $\mathrm{H}$ (curl)-elliptic QVI and prove its existence using a fixed-point argument in combination with techniques from variational inequalities and Maxwell's equations. Based on the existence result for the $\mathrm{H}$ (curl)-elliptic QVI, we examine the stability and convergence of the Euler scheme, which serve as our fundament for the global well-posedness of the governing hyperbolic Maxwell QVI.
\end{abstract}

Mathematics Subject Classification. 35L85, 35Q60.

Received January 13, 2021. Accepted June 15, 2021.

\section{INTRODUCTION}

Ever since the discovery of superconductivity by Heike Kamerlingh Onnes in 1911, various modern applications and key technologies have been developed. Among many other profound applications, we mention magnetic resonance imaging, magnetic confinement fusion, and magnetic levitation. Such technological advances are made possible by superconductors due to their fundamental properties of vanishing electrical resistance and expulsion of applied magnetic fields (Meissner effect) occurring when the temperature is cooled below the critical temperature. A prominent critical-state model describing the irreversible magnetization process in high-temperature superconductivity was proposed by Bean $[5,6]$. His model postulates a nonlinear and non-smooth constitutive relation between the current density and the electric field through the so-called critical current as follows:

(B1) the current density strength $|\boldsymbol{J}|$ cannot exceed the critical current $j_{c}$

(B2) the electric field $\boldsymbol{E}$ vanishes if $|\boldsymbol{J}|$ is strictly less than $j_{c}$

(B3) the electric field $\boldsymbol{E}$ is parallel to the current density $\boldsymbol{J}$.

Shortly after the publication by Bean [5], Kim et al. [13] revealed and reported on the magnetic field dependency in the critical current density $j_{c}=j_{c}(\boldsymbol{H})$.

The Bean-Kim model governed by the eddy current equations leads to a parabolic quasi-variational inequality (QVI). Prigozhin [20] was the first to introduce this formulation. Barrett and Prigozhin [3,4] examined the

Keywords and phrases. Maxwell quasi-variational inequality, well-posedness, Bean-Kim critical-state model, high-temperature superconductivity.

Universität Duisburg-Essen, Fakultät für Mathematik, Thea-Leymann-Str. 9, D-45127 Essen, Germany.

* Corresponding author: irwin. yousept@uni-due.de 
TABLE 1. Critical temperature $\theta_{c}$ for several high-temperature superconductors.

\begin{tabular}{lll}
\hline \hline $\mathrm{Ba}-\mathrm{La}-\mathrm{Cu}-\mathrm{O}$ & $\mathrm{Y}-\mathrm{Ba}-\mathrm{Cu}-\mathrm{O}$ & $\mathrm{Hg}-\mathrm{Ba}-\mathrm{Ca}-\mathrm{Cu}-\mathrm{O}$ \\
\hline $35 \mathrm{~K} /-238.15^{\circ} \mathrm{C}$ & $93 \mathrm{~K} /-180.15^{\circ} \mathrm{C}$ & $133 \mathrm{~K} /-140.15^{\circ} \mathrm{C}$ \\
\hline
\end{tabular}

associated QVI in a scalar two-dimensional (2D) setting and its dual formulation. For the analysis of general parabolic and elliptic QVI problems with gradient and curl constraints, we refer to Rodrigues and Santos [21,22] and Miranda et al. [16]. All these contributions take into account the eddy current approximation of the full Maxwell formulation leading to problems with a parabolic character. The analysis of Bean's critical-state model with displacement current goes back to [12].

This paper is a continuation of the recent papers [32,33] on hyperbolic Maxwell variational inequalities (VI), including those arising in high-temperature superconductivity and electromagnetic shielding $(c f .[14,27,28])$. The goal of the present paper is to explore hyperbolic Maxwell QVI arising from the Bean-Kim model (B1)-(B3) with magnetic field and temperature dependence in the critical current $j_{c}=j_{c}(\theta, \boldsymbol{H})$. In particular, temperature effects are included due to its central importance in the superconductivity phenomena. As reported in [2], the temperature dependence in the critical current of the $\mathrm{Y}-\mathrm{Ba}-\mathrm{Cu}-\mathrm{O}$ bulk superconductor exhibits a continuous and piecewise smooth structure ([2], Fig. 2). More precisely, it features a linear behaviour of the type $\left(\theta_{c}-\theta\right)$, if the temperature $\theta$ is sufficiently smaller than $\theta_{c}$ (see Tab. 1). If $\theta$ is close to $\theta_{c}$, then a nonlinear behaviour of the type $\left(1-\frac{\theta}{\theta_{c}}\right)^{3 / 2}$ is observed, and the critical current vanishes if $\theta \geq \theta_{c}$. This behaviour is in agreement with the theoretical model of granular superconductors [8]. Similarly, Deutscher and Müller [9] reported on a temperature dependence of the type $\left(1-\frac{\theta}{\theta_{c}}\right)^{2}$ in the case of $\theta \approx \theta_{c}$ for the critical currents of high-temperature oxides.

Let $T \in \mathbb{R}^{+}$and $\Omega \subset \mathbb{R}^{3}$ be a bounded Lipschitz domain filled with a high-temperature superconductor. Considering the full Maxwell formulation for the electromagnetic fields, the Bean-Kim model (B1)-(B3) with $j_{c}=j_{c}(\theta, \boldsymbol{H})$ leads to the following problem:

$$
\begin{cases}\epsilon \partial_{t} \boldsymbol{E}-\operatorname{curl} \boldsymbol{H}+\boldsymbol{J}=\boldsymbol{u} & \text { in } \Omega \times(0, T) \\ \mu \partial_{t} \boldsymbol{H}+\operatorname{curl} \boldsymbol{E}=0 & \text { in } \Omega \times(0, T) \\ \boldsymbol{E} \times \boldsymbol{n}=0 & \text { on } \partial \Omega \times(0, T) \\ \boldsymbol{E}(\cdot, 0)=\boldsymbol{E}_{0} \quad \boldsymbol{H}(\cdot, 0)=\boldsymbol{H}_{0} & \text { in } \Omega \\ |\boldsymbol{J}(x, t)| \leq j_{c}(x, \theta(x, t), \boldsymbol{H}(x, t)) & \text { for a.e. }(x, t) \in \Omega \times(0, T) \\ \boldsymbol{J}(x, t) \cdot \boldsymbol{E}(x, t)=j_{c}(x, \theta(x, t), \boldsymbol{H}(x, t))|\boldsymbol{E}(x, t)| & \text { for a.e. }(x, t) \in \Omega \times(0, T) .\end{cases}
$$

All assumptions for the data involved in (1.1) are summarized in Assumptions 2.1 and 4.1. In particular, motivated from the experimental measurements $[2,8,9]$, our analysis relies on local boundedness and local Lipschitz continuity assumptions for the critical current with respect to the temperature. On the other hand, the magnetic field dependency in $j_{c}$ is assumed to be globally bounded and Lipschitz continuous. This stronger assumption ( $c f$. [7] for physical measurement supporting this assumption) seems to be indispensable in order to handle the notorious hyperbolic QVI-character of (1.1). More precisely, the variational formulation for (1.1) (see Cor. 5.2) is given by the following hyperbolic Maxwell QVI: Find $(\boldsymbol{E}, \boldsymbol{H}) \in W^{1, \infty}\left((0, T), \boldsymbol{L}^{2}(\Omega)\right) \cap L^{\infty}\left((0, T), \boldsymbol{H}_{0}(\mathbf{c u r l})\right) \times W^{1, \infty}\left((0, T), \boldsymbol{L}^{2}(\Omega)\right) \cap L^{\infty}((0, T), \boldsymbol{X}(\Omega))$ such that 


$$
\left\{\begin{array}{l}
\int_{\Omega} \epsilon \partial_{t} \boldsymbol{E}(t) \cdot(\boldsymbol{v}-\boldsymbol{E}(t))+\mu \partial_{t} \boldsymbol{H}(t) \cdot(\boldsymbol{w}-\boldsymbol{H}(t))+\operatorname{curl} \boldsymbol{E}(t) \cdot \boldsymbol{w}-\operatorname{curl} \boldsymbol{H}(t) \cdot \boldsymbol{v} \mathrm{d} x \\
+\int_{\Omega} j_{c}(x, \theta(x, t), \boldsymbol{H}(x, t))(|\boldsymbol{v}(x)|-|\boldsymbol{E}(x, t)|) \mathrm{d} x \geq \int_{\Omega} \boldsymbol{u}(t) \cdot(\boldsymbol{v}-\boldsymbol{E}(t)) \mathrm{d} x \\
\text { for a.e. } t \in(0, T) \text { and all }(\boldsymbol{v}, \boldsymbol{w}) \in \boldsymbol{L}^{2}(\Omega) \times \boldsymbol{L}^{2}(\Omega) \\
(\boldsymbol{E}, \boldsymbol{H})(0)=\left(\boldsymbol{E}_{0}, \boldsymbol{H}_{0}\right) .
\end{array}\right.
$$

We note that the time derivatives $\partial_{t} \boldsymbol{E}$ and $\partial_{t} \boldsymbol{H}$ are of class $L^{\infty}\left((0, T), \boldsymbol{L}^{2}(\Omega)\right)$. Thus, for a.e. $t \in(0, T)$, both $\partial_{t} \boldsymbol{E}(t)$ and $\partial_{t} \boldsymbol{H}(t)$ are $\boldsymbol{L}^{2}(\Omega)$-functions, and so the multiplication dot $\partial_{t} \boldsymbol{E}(t) \cdot \boldsymbol{v}\left(\right.$ resp. $\left.\partial_{t} \boldsymbol{H}(t) \cdot \boldsymbol{w}\right)$ in $(\mathrm{EQVI})$ is nothing but $\sum_{j=1}^{3}\left(\partial_{t} \boldsymbol{E}(t)\right)_{j} \boldsymbol{v}_{j}$ (resp. $\left.\sum_{j=1}^{3}\left(\partial_{t} \boldsymbol{H}(t)\right)_{j} \boldsymbol{w}_{j}\right)$.

In the case of $j_{c}=j_{c}(x)$, (QVI) simplifies to a VI problem, which was originally introduced and discussed in [30] ( $c f$. also [32] for a more general class of hyperbolic Maxwell VI). The pure temperature dependence $j_{c}=j_{c}(x, \theta(x, t))$ was considered in the subsequent paper [27] focusing on its fully discrete analysis. The present paper extends [27,30]: We consider the more realistic case $j_{c}=j_{c}(x, \theta(x, t), \boldsymbol{H}(x, t))$ with less regularity requirement for the data $\boldsymbol{u}, \theta$, and $\left(\boldsymbol{E}_{0}, \boldsymbol{H}_{0}\right)$. Note that, since our problem features a QVI character, the prior developed VI-techniques in $[27,30,32]$ cannot be directly applied to (QVI) and require certain extensions.

The first part of this paper is devoted to the following nonlinear PDE-system:

$$
\begin{cases}\epsilon \boldsymbol{e}-\operatorname{curl} \boldsymbol{h}+\boldsymbol{j}=\mathbf{f} & \text { in } \Omega \\ \mu \boldsymbol{h}+\operatorname{curl} \boldsymbol{e}=\mu \mathbf{g} & \text { in } \Omega \\ \boldsymbol{e} \times \boldsymbol{n}=0 & \text { on } \partial \Omega \\ |\boldsymbol{j}(x)| \leq j_{c}(x, y(x), \boldsymbol{h}(x)) & \text { for a.e. } x \in \Omega \\ \boldsymbol{j}(x) \cdot \boldsymbol{e}(x)=j_{c}(x, y(x), \boldsymbol{h}(x))|\boldsymbol{e}(x)| & \text { for a.e. } x \in \Omega\end{cases}
$$

where $\mathbf{f}, \mathbf{g}$, and $y$ are given data. The variational formulation of (1.2) in terms of $\boldsymbol{e}$ (see Cor. 3.3) is given by the following $\boldsymbol{H}$ (curl)-elliptic QVI: Find $\boldsymbol{e} \in \boldsymbol{H}_{0}(\mathbf{c u r l})$ such that

$$
\begin{array}{r}
a(\boldsymbol{e}, \boldsymbol{v}-\boldsymbol{e})+\int_{\Omega} j_{c}\left(x, y(x), \mathbf{g}(x)-\mu^{-1}(x) \operatorname{curl} \boldsymbol{e}(x)\right)(|\boldsymbol{v}(x)|-|\boldsymbol{e}(x)|) \mathrm{d} x \\
\geq(\mathbf{f}+\operatorname{curl} \mathbf{g}, \boldsymbol{v}-\boldsymbol{e})_{\boldsymbol{L}^{2}(\Omega)} \quad \forall \boldsymbol{v} \in \boldsymbol{H}_{0}(\mathbf{c u r l}),
\end{array}
$$

where the bilinear form $a: \boldsymbol{H}_{0}(\mathbf{c u r l}) \times \boldsymbol{H}_{0}(\mathbf{c u r l}) \rightarrow \mathbb{R}$ is defined as in (3.3). To derive an existence result for (1.2), we first drop out the magnetic field dependency in the critical current $j_{c}$. This leads to a complementaritytype problem, which we shall study through the theory of variational inequalities. Then, on the basis of the proposed complementarity-type problem, we formulate (1.2) as a fixed-point problem and show its existence (Thm. 3.2) by means of the Maxwell compactness embedding theory $[19,26]$ along with the Schauder fixed-point theorem.

After deriving a well-posedness result for (1.2), we consider the time-discrete problem $\left(\mathrm{P}_{n}\right)$ associated with (1.1) based on the implicit Euler scheme (Rothe method). While the existence of $\left(\mathrm{P}_{n}\right)$ is covered by the developed existence result for (1.2), the uniqueness is obtained if the time step is sufficiently small. We investigate the stability analysis of the resulting time-discrete magnetic and electric fields, including their difference quotients (Lems. 4.5 and 4.6). Differently from Assumption 2.2 of [27], our stability analysis does not rely on any compatibility condition for the initial data. We circumvent this issue by introducing an auxiliary current density (4.2) and initial difference quotients (4.3), that preserve the pivotal QVI structure at the initial time (4.4). This construction allows us to prove stability and convergence of $\left(\mathrm{P}_{n}\right)$ leading to our final result (Cor. 5.2) on the well-posedness for (QVI) for all $\boldsymbol{u} \in H^{1}\left((0, T), \boldsymbol{L}^{2}(\Omega)\right), \theta \in H^{1}\left((0, T), L^{2}(\Omega)\right) \cap \mathcal{C}\left([0, T], L^{\infty}(\Omega)\right)$, and $\left(\boldsymbol{E}_{0}, \boldsymbol{H}_{0}\right) \in \boldsymbol{H}_{0}(\mathbf{c u r l}) \times \boldsymbol{X}(\Omega)$ without any compatibility assumption. 


\section{Preliminaries}

For a given Hilbert space $V$, we use the notation $\|\cdot\|_{V}$ and $(\cdot, \cdot)_{V}$ for a standard norm and a standard scalar product in $V$. By $V^{*}$ we denote the dual space of $V$. If $V$ is continuously embedded in another normed linear space $Y$, then we write $V \hookrightarrow Y$ for the associated injection. A bold typeface is used to indicate a threedimensional vector function or a Hilbert space of three-dimensional vector functions. The main Hilbert space for our analysis is

$$
\boldsymbol{H}(\operatorname{curl}):=\left\{\boldsymbol{q} \in \boldsymbol{L}^{2}(\Omega) \mid \operatorname{curl} \boldsymbol{q} \in \boldsymbol{L}^{2}(\Omega)\right\},
$$

where the curl-operator is understood in the sense of distributions. As usual, $\mathcal{C}_{0}^{\infty}(\Omega)$ stands for the space of all infinitely differentiable three-dimensional vector functions with compact support contained in $\Omega$. We denote the closure of $\mathcal{C}_{0}^{\infty}(\Omega)$ with respect to the $\boldsymbol{H}(\mathbf{c u r l})$-topology by $\boldsymbol{H}_{0}(\mathbf{c u r l})$. It is well-known that the Hilbert space $\boldsymbol{H}_{0}(\mathbf{c u r l})$ admits the following characterization ( $c f$. [32], Appendix A):

$$
\begin{aligned}
\boldsymbol{H}_{0}(\operatorname{curl}) & =\left\{\boldsymbol{q} \in \boldsymbol{H}(\operatorname{curl}) \mid(\operatorname{curl} \boldsymbol{q}, \boldsymbol{v})_{\boldsymbol{L}^{2}(\Omega)}=(\boldsymbol{q}, \operatorname{curl} \boldsymbol{v})_{\boldsymbol{L}^{2}(\Omega)} \quad \forall \boldsymbol{v} \in \boldsymbol{H}(\operatorname{curl})\right\} \\
& =\left\{\boldsymbol{q} \in \boldsymbol{H}(\operatorname{curl}) \mid \gamma_{t}(\boldsymbol{q})=0\right\},
\end{aligned}
$$

where $\gamma_{t}: \boldsymbol{H}(\mathbf{c u r l}) \rightarrow \boldsymbol{H}^{-\frac{1}{2}}(\partial \Omega)$ denotes the tangential trace $\left(c f\right.$. [11], Thm. 2.11). We note that $\gamma_{t}(\boldsymbol{q})=0$ generalizes the boundary condition $\boldsymbol{q} \times \boldsymbol{n}=0$ on $\partial \Omega$. Another important Hilbert space used in our analysis is

$$
\boldsymbol{X}(\Omega):=\left\{\boldsymbol{q} \in \boldsymbol{H}(\mathbf{c u r l}) \mid(\mu \boldsymbol{q}, \nabla \phi)_{\boldsymbol{L}^{2}(\Omega)}=0 \quad \forall \phi \in H^{1}(\Omega)\right\} .
$$

For an almost everywhere positive function $\alpha \in L^{\infty}(\Omega)$, we use the notation $\boldsymbol{L}_{\alpha}^{2}(\Omega)$ for the weighted $\boldsymbol{L}^{2}(\Omega)$-space endowed with the weighted scalar product $(\alpha \cdot, \cdot)_{L^{2}(\Omega)}$.

Let us now formulate the required regularity assumption on the electric permittivity $\epsilon: \Omega \rightarrow(0, \infty)$, the magnetic permeability $\mu: \Omega \rightarrow(0, \infty)$, and the critical current $j_{c}: \Omega \times \mathbb{R} \times \mathbb{R}^{3} \rightarrow[0, \infty)$.

Assumption 2.1 (Regularity assumption on the material parameters).

(A1) We suppose that $\epsilon, \mu \in L^{\infty}(\Omega)$ and there exist positive constants $0<\underline{\epsilon} \leq \bar{\epsilon}$ and $0<\underline{\mu} \leq \bar{\mu}$ such that

$$
\underline{\epsilon} \leq \epsilon(x) \leq \bar{\epsilon} \quad \text { for a.e. } x \in \Omega \quad \text { and } \quad \underline{\mu} \leq \mu(x) \leq \bar{\mu} \text { for a.e. } x \in \Omega .
$$

(A2) For every fixed $(y, h) \in \mathbb{R} \times \mathbb{R}^{3}, j_{c}(\cdot, y, h): \Omega \rightarrow \mathbb{R}$ is Lebesgue measurable.

(A3) For every $M>0$, there exists a constant $C(M)>0$ such that

$$
0 \leq j_{c}(x, y, h) \leq C(M)
$$

for a.e. $x \in \Omega$ and all $(y, h) \in[-M, M] \times \mathbb{R}^{3}$.

(A4) For every $M>0$, there exists a constant $L(M)>0$ such that

$$
\left|j_{c}\left(x, y_{1}, h_{1}\right)-j_{c}\left(x, y_{2}, h_{2}\right)\right| \leq L(M)\left(\left|y_{1}-y_{2}\right|+\left|h_{1}-h_{2}\right|\right)
$$

for a.e. $x \in \Omega$ and all $\left(y_{1}, h_{1}\right),\left(y_{2}, h_{2}\right) \in[-M, M] \times \mathbb{R}^{3}$.

Note that (A3) and (A4) require the global boundedness and the global Lipschitz continuity of $j_{c}: \Omega \times$ $\mathbb{R} \times \mathbb{R}^{3} \rightarrow[0, \infty)$ with respect to the third component (magnetic field). An example for the magnetic field dependency satisfying (A3) and (A4) is

$$
h \mapsto c_{1}\left(c_{2}+c_{3}|h|^{s}\right)^{-1}
$$

for some positive constants $c_{1}, c_{2}, c_{3}>0$ and some exponent $s>1$. The mapping (2.3) is obviously globally bounded. Furthermore, the Lipschitz continuity holds true as its derivative is globally bounded. As confirmed by physical measurement [7], such a model (2.3) is reasonable for describing the magnetic field dependency in critical currents of certain superconductors. 
Remark 2.2. Our analysis can be extended to the case where the critical current explicitly depends on the time variable, i.e., $j_{c}: \Omega \times[0, T] \times \mathbb{R} \times \mathbb{R}^{3} \rightarrow[0, \infty)$. In this case, for every fixed $(t, y, h) \in[0, T] \times \mathbb{R} \times \mathbb{R}^{3}$, the function $j_{c}(\cdot, t, y, h): \Omega \rightarrow \mathbb{R}$ is assumed to be Lebesgue measurable. Moreover, we replace (A3)-(A4) by

$$
\begin{aligned}
0 & \leq j_{c}(x, t, y, h) \leq C(M) \\
\left|j_{c}\left(x, t_{1}, y_{1}, h_{1}\right)-j_{c}\left(x, t_{2}, y_{2}, h_{2}\right)\right| & \leq L(M)\left(\left|t_{1}-t_{2}\right|+\left|y_{1}-y_{2}\right|+\left|h_{1}-h_{2}\right|\right)
\end{aligned}
$$

for a.e. $x \in \Omega$ and all $(t, y, h),\left(t_{1}, y_{1}, h_{1}\right),\left(t_{2}, y_{2}, h_{2}\right) \in[0, T] \times[-M, M] \times \mathbb{R}^{3}$.

\section{WELL-POSEDNESS FOR (EQVI)}

We start our investigation by examining the following complementarity-type problem.

Lemma 3.1. Let Assumption 2.1 be satisfied. Then, for every $y \in L^{\infty}(\Omega)$ and $(\mathbf{f}, \mathbf{g}, \boldsymbol{z}) \in \boldsymbol{L}^{2}(\Omega) \times \boldsymbol{X}(\Omega) \times \boldsymbol{L}^{2}(\Omega)$, the complementarity-type problem

$$
\begin{cases}\epsilon \boldsymbol{e}-\operatorname{curl} \boldsymbol{h}+\boldsymbol{j}=\mathbf{f} & \text { in } \Omega \\ \mu \boldsymbol{h}+\operatorname{curl} \boldsymbol{e}=\mu \mathrm{g} & \text { in } \Omega \\ \boldsymbol{e} \times \boldsymbol{n}=0 & \text { on } \partial \Omega \\ |\boldsymbol{j}(x)| \leq j_{c}(x, y(x), \boldsymbol{z}(x)) & \text { for a.e. } x \in \Omega \\ \boldsymbol{j}(x) \cdot \boldsymbol{e}(x)=j_{c}(x, y(x), \boldsymbol{z}(x))|\boldsymbol{e}(x)| & \text { for a.e. } x \in \Omega\end{cases}
$$

admits a unique solution $(\boldsymbol{e}, \boldsymbol{h}, \boldsymbol{j}) \in \boldsymbol{H}_{0}(\mathbf{c u r l}) \times \boldsymbol{X}(\Omega) \times \boldsymbol{L}^{\infty}(\Omega)$ satisfying

$$
\|\boldsymbol{h}\|_{\boldsymbol{X}(\Omega)} \leq \bar{\epsilon}^{\frac{1}{2}}\|\mathbf{f}\|_{L_{\frac{1}{\epsilon}}^{2}(\Omega)}+\underline{\mu}^{-\frac{1}{2}}\|\mathbf{g}\|_{\boldsymbol{L}_{\mu}^{2}(\Omega)}+\left(\underline{\mu}^{-\frac{1}{2}}+\bar{\epsilon}^{\frac{1}{2}}\right)\|\mathbf{f}+\operatorname{curl} \mathbf{g}\|_{L_{\frac{1}{\epsilon}}^{2}(\Omega)}+\sqrt{\frac{\bar{\epsilon}}{\underline{\epsilon}}} C\left(\|y\|_{L^{\infty}(\Omega)}\right)|\Omega|^{\frac{1}{2}} .
$$

Proof. Let $y \in L^{\infty}(\Omega)$ and $(\mathbf{f}, \mathbf{g}, \boldsymbol{z}) \in \boldsymbol{L}^{2}(\Omega) \times \boldsymbol{X}(\Omega) \times \boldsymbol{L}^{2}(\Omega)$. We introduce a continuous and coercive bilinear form

$$
a: \boldsymbol{H}_{0}(\operatorname{curl}) \times \boldsymbol{H}_{0}(\operatorname{curl}) \rightarrow \mathbb{R}, \quad a(\boldsymbol{u}, \boldsymbol{v}):=\left(\mu^{-1} \operatorname{curl} \boldsymbol{u}, \operatorname{curl} \boldsymbol{v}\right)_{\boldsymbol{L}^{2}(\Omega)}+(\epsilon \boldsymbol{u}, \boldsymbol{v})_{\boldsymbol{L}^{2}(\Omega)},
$$

and a convex and continuous functional

$$
\Psi: \boldsymbol{L}^{2}(\Omega) \rightarrow \mathbb{R}, \quad \Psi(\boldsymbol{v}):=\int_{\Omega} j_{c}(x, y(x), \boldsymbol{z}(x))|\boldsymbol{v}(x)| \mathrm{d} x .
$$

The classical result [15] implies that the variational inequality

$$
a(\boldsymbol{e}, \boldsymbol{v}-\boldsymbol{e})+\Psi(\boldsymbol{v})-\Psi(\boldsymbol{e}) \geq(\mathbf{f}+\operatorname{curl} \mathbf{g}, \boldsymbol{v}-\boldsymbol{e})_{\boldsymbol{L}^{2}(\Omega)} \quad \forall \boldsymbol{v} \in \boldsymbol{H}_{0}(\mathbf{c u r l})
$$

admits a unique solution $\boldsymbol{e} \in \boldsymbol{H}_{0}(\mathbf{c u r l})$. Setting $\boldsymbol{v}=0$ and $\boldsymbol{v}=2 \boldsymbol{e}$ in (3.4), we obtain

$$
a(\boldsymbol{e}, \boldsymbol{e})+\Psi(\boldsymbol{e})=(\mathbf{f}+\operatorname{curl} \mathbf{g}, \boldsymbol{e})_{\boldsymbol{L}^{2}(\Omega)} .
$$

Applying this identity to (3.4) implies

$$
\Psi(\boldsymbol{v}) \geq L(\boldsymbol{v}):=(\mathbf{f}+\operatorname{curl} \mathbf{g}, \boldsymbol{v})_{\boldsymbol{L}^{2}(\Omega)}-a(\boldsymbol{e}, \boldsymbol{v}) \quad \forall \boldsymbol{v} \in \boldsymbol{H}_{0}(\mathbf{c u r l}) .
$$

Therefore, as $L: \boldsymbol{H}_{0}(\mathbf{c u r l}) \rightarrow \mathbb{R}$ is linear and $\Psi: \boldsymbol{L}^{2}(\Omega) \rightarrow \mathbb{R}$ is sublinear, i.e.,

$$
\Psi(\boldsymbol{v}+\boldsymbol{w}) \leq \Psi(\boldsymbol{v})+\Psi(\boldsymbol{w}), \quad \Psi(t \boldsymbol{v})=t \Psi(\boldsymbol{v}) \quad \forall \boldsymbol{v}, \boldsymbol{w} \in \boldsymbol{L}^{2}(\Omega), \quad \forall t \geq 0,
$$

the Hahn-Banach theorem ([23], Thm. 3.2) yields the existence of $\boldsymbol{j} \in \boldsymbol{L}^{2}(\Omega)$ such that

$$
(\boldsymbol{j}, \boldsymbol{v})_{\boldsymbol{L}^{2}(\Omega)}=L(\boldsymbol{v}) \quad \forall \boldsymbol{v} \in \boldsymbol{H}_{0}(\operatorname{curl})
$$




$$
(\boldsymbol{j}, \boldsymbol{v})_{\boldsymbol{L}^{2}(\Omega)} \leq \Psi(\boldsymbol{v}) \quad \forall \boldsymbol{v} \in \boldsymbol{L}^{2}(\Omega)
$$

According to the definition, equation (3.7) is equivalent to

$$
\left(\mu^{-1} \operatorname{curl} \boldsymbol{e}, \operatorname{curl} \boldsymbol{v}\right)_{\boldsymbol{L}^{2}(\Omega)}=(\mathbf{f}+\operatorname{curl} g-\boldsymbol{j}-\epsilon \boldsymbol{e}, \boldsymbol{v}) \quad \forall \boldsymbol{v} \in \boldsymbol{H}_{0}(\operatorname{curl}) .
$$

Consequently, the distributional definition of the curl-operator yields that

$$
\mu^{-1} \operatorname{curl} \boldsymbol{e} \in \boldsymbol{H}(\operatorname{curl}) \quad \text { with } \quad \operatorname{curl} \mu^{-1} \operatorname{curl} \boldsymbol{e}=\mathbf{f}+\operatorname{curl} g-\boldsymbol{j}-\epsilon \boldsymbol{e} .
$$

Together with

$$
\left(\mu\left(\mu^{-1} \operatorname{curl} e\right), \nabla \phi\right)_{L^{2}(\Omega)} \underbrace{=}_{(2.1)}(e, \operatorname{curl} \nabla \phi)_{L^{2}(\Omega)}=0 \quad \forall \phi \in H^{1}(\Omega),
$$

equation (3.10) implies $\mu^{-1}$ curl $e \in \boldsymbol{X}(\Omega)$. Thus, defining $\boldsymbol{h}:=\mathrm{g}-\mu^{-1} \operatorname{curl} \boldsymbol{e} \in \boldsymbol{X}(\Omega)$, it follows immediately from (3.10) that $\epsilon \boldsymbol{e}-\mathbf{c u r l} \boldsymbol{h}+\boldsymbol{j}=\mathbf{f}$. Next, let us show that

$$
|\boldsymbol{j}(x)| \leq j_{c}(x, y(x), \boldsymbol{z}(x)) \text { for a.e. } x \in \Omega .
$$

Suppose that there exists a Lebesgue measurable set $\omega \subset \Omega$ of non-zero measure such that $j_{c}(x, y(x), \boldsymbol{z}(x))<$ $|\boldsymbol{j}(x)|$ for a.e. $x \in \omega$. Then setting $\boldsymbol{v}_{\omega}:=\frac{\boldsymbol{j}}{|\boldsymbol{j}|} \chi_{\omega} \in \boldsymbol{L}^{2}(\Omega)$ in (3.8), where $\chi_{\omega}$ denotes the characteristic function of $\omega$, leads to a contradiction

$$
\int_{\omega} j_{c}(x, y(x), \boldsymbol{z}(x)) \mathrm{d} x<\int_{\omega}|\boldsymbol{j}(x)| \mathrm{d} x=\left(\boldsymbol{j}, \boldsymbol{v}_{\omega}\right)_{\boldsymbol{L}^{2}(\Omega)} \leq \Psi\left(\boldsymbol{v}_{\omega}\right)=\int_{\omega} j_{c}(x, y(x), \boldsymbol{z}(x)) \mathrm{d} x .
$$

Consequently, the inequality (3.11) is valid. In particular, the properties (3.11) and (A3) with $h=\boldsymbol{z}(x)$ yield that

$$
|\boldsymbol{j}(x)| \leq j_{c}(x, y(x), \boldsymbol{z}(x)) \leq C\left(\|y\|_{L^{\infty}(\Omega)}\right) \quad \text { for a.e. } x \in \Omega,
$$

and so $\boldsymbol{j} \in \boldsymbol{L}^{\infty}(\Omega)$. Inserting now $\boldsymbol{v}=\boldsymbol{e}$ in (3.9) and taking (3.5) into account, it follows that

$$
0=\int_{\Omega} j_{c}(x, y(x), \boldsymbol{z}(x))|\boldsymbol{e}(x)|-\boldsymbol{j}(x) \cdot \boldsymbol{e}(x) \mathrm{d} x .
$$

As a result of (3.11) and (3.12), we obtain

$$
\boldsymbol{j}(x) \cdot \boldsymbol{e}(x)=j_{c}(x, y(x), \boldsymbol{z}(x))|\boldsymbol{e}(x)| \quad \text { for a.e. } x \in \Omega .
$$

In conclusion, the triple $(\boldsymbol{e}, \boldsymbol{h}, \boldsymbol{j}) \in \boldsymbol{H}_{0}(\mathbf{c u r l}) \times \boldsymbol{X}(\Omega) \times \boldsymbol{L}^{\infty}(\Omega)$ is a solution of (3.1).

Let us prove the a priori estimate (3.2). First, equation (3.5) implies

$$
\left\|\epsilon^{\frac{1}{2}} \boldsymbol{e}\right\|_{\boldsymbol{L}^{2}(\Omega)}^{2}+\left\|\mu^{-\frac{1}{2}} \operatorname{curl} \boldsymbol{e}\right\|_{\boldsymbol{L}^{2}(\Omega)}^{2} \leq\left\|\epsilon^{-\frac{1}{2}}(\mathbf{f}+\operatorname{curl} \mathbf{g})\right\|_{\boldsymbol{L}^{2}(\Omega)}^{2},
$$

from which it follows that

$$
\left\|\mu^{\frac{1}{2}} \boldsymbol{h}\right\|_{\boldsymbol{L}^{2}(\Omega)}=\left\|\mu^{\frac{1}{2}} \mathbf{g}-\mu^{-\frac{1}{2}} \operatorname{curl} \boldsymbol{e}\right\|_{\boldsymbol{L}^{2}(\Omega)} \leq\left\|\mu^{\frac{1}{2}} \mathbf{g}\right\|_{\boldsymbol{L}^{2}(\Omega)}+\left\|\epsilon^{-\frac{1}{2}}(\mathbf{f}+\operatorname{curl} \mathbf{g})\right\|_{\boldsymbol{L}^{2}(\Omega)} .
$$

Also, the inequalities (3.13) and (3.11) together with (A3) imply

$$
\begin{aligned}
\left\|\epsilon^{-\frac{1}{2}} \operatorname{curl} \boldsymbol{h}\right\|_{\boldsymbol{L}^{2}(\Omega)} & =\left\|\epsilon^{\frac{1}{2}} \boldsymbol{e}+\epsilon^{-\frac{1}{2}}(\boldsymbol{j}-\mathbf{f})\right\|_{\boldsymbol{L}^{2}(\Omega)} \\
& \leq\left\|\epsilon^{-\frac{1}{2}}(\mathbf{f}+\mathbf{c u r l} \mathbf{g})\right\|_{\boldsymbol{L}^{2}(\Omega)}+\underline{\epsilon}^{-\frac{1}{2}} C\left(\|y\|_{L^{\infty}(\Omega)}\right)|\Omega|^{\frac{1}{2}}+\left\|\epsilon^{-\frac{1}{2}} \mathbf{f}\right\|_{\boldsymbol{L}^{2}(\Omega)} .
\end{aligned}
$$


From (3.14) and (3.15), we come to the conclusion that

$$
\begin{aligned}
& \|\boldsymbol{h}\|_{\boldsymbol{X}(\Omega)} \\
& =\|\boldsymbol{h}\|_{\boldsymbol{H}(\mathbf{c u r l})}=\sqrt{\left\|\mu^{-\frac{1}{2}} \mu^{\frac{1}{2}} \boldsymbol{h}\right\|_{\boldsymbol{L}^{2}(\Omega)}^{2}+\left\|\epsilon^{\frac{1}{2}} \epsilon^{-\frac{1}{2}} \operatorname{curl} \boldsymbol{h}\right\|_{\boldsymbol{L}^{2}(\Omega)}^{2}} \\
& \leq \underline{\mu}^{-\frac{1}{2}}\left\|\mu^{\frac{1}{2}} \boldsymbol{h}\right\|_{\boldsymbol{L}^{2}(\Omega)}+\bar{\epsilon}^{\frac{1}{2}}\left\|\epsilon^{-\frac{1}{2}} \mathbf{c u r l} \boldsymbol{h}\right\|_{\boldsymbol{L}^{2}(\Omega)} \\
& \leq \underline{\mu}^{-\frac{1}{2}}\left\|\mu^{\frac{1}{2}} \mathbf{g}\right\|_{\boldsymbol{L}^{2}(\Omega)}+\left(\underline{\mu}^{-\frac{1}{2}}+\bar{\epsilon}^{\frac{1}{2}}\right)\left\|\epsilon^{-\frac{1}{2}}(\mathbf{f}+\operatorname{curl} \mathbf{g})\right\|_{\boldsymbol{L}^{2}(\Omega)}+\bar{\epsilon}^{\frac{1}{2}}\left(\underline{\epsilon}^{-\frac{1}{2}} C\left(\|y\|_{L^{\infty}(\Omega)}\right)|\Omega|^{\frac{1}{2}}+\left\|\epsilon^{-\frac{1}{2}} \mathbf{f}\right\|_{\boldsymbol{L}^{2}(\Omega)}\right) .
\end{aligned}
$$

Uniqueness. Suppose that $(\widetilde{\boldsymbol{e}}, \widetilde{\boldsymbol{h}}, \widetilde{\boldsymbol{j}}) \in \boldsymbol{H}_{0}(\mathbf{c u r l}) \times \boldsymbol{X}(\Omega) \times \boldsymbol{L}^{\infty}(\Omega)$ is another solution of (3.1), i.e., it satisfies

$$
\begin{aligned}
\epsilon \widetilde{\boldsymbol{e}}-\operatorname{curl} \widetilde{\boldsymbol{h}}+\widetilde{\boldsymbol{j}} & =\mathbf{f} & & \\
\mu \widetilde{\boldsymbol{h}}+\operatorname{curl} \widetilde{\boldsymbol{e}} & =\mu \mathbf{g} & & \\
|\widetilde{\boldsymbol{j}}(x)| & \leq j_{c}(x, y(x), \boldsymbol{z}(x)) & & \text { for a.e. } x \in \Omega \\
\widetilde{\boldsymbol{j}}(x) \cdot \widetilde{\boldsymbol{e}}(x) & =j_{c}(x, y(x), \boldsymbol{z}(x))|\widetilde{\boldsymbol{e}}(x)| & & \text { for a.e. } x \in \Omega .
\end{aligned}
$$

From (3.16) and (3.17) together with (2.1), we obtain that

$$
a(\widetilde{\boldsymbol{e}}, \boldsymbol{v}-\widetilde{\boldsymbol{e}})+(\widetilde{\boldsymbol{j}}, \boldsymbol{v}-\widetilde{\boldsymbol{e}})_{\boldsymbol{L}^{2}(\Omega)}=(\mathbf{f}+\operatorname{curlg}, \boldsymbol{v}-\widetilde{\boldsymbol{e}})_{\boldsymbol{L}^{2}(\Omega)} \quad \forall \boldsymbol{v} \in \boldsymbol{H}_{0}(\operatorname{curl}) .
$$

On the other hand, the properties (3.18) and (3.19) imply

$$
\int_{\Omega} \widetilde{\boldsymbol{j}}(x) \cdot(\boldsymbol{v}(x)-\widetilde{\boldsymbol{e}}(x)) \mathrm{d} x \leq \int_{\Omega} j_{c}(x, y(x), \boldsymbol{z}(x))(|\boldsymbol{v}(x)|-|\widetilde{\boldsymbol{e}}(x)|) \mathrm{d} x=\Psi(\boldsymbol{v})-\Psi(\widetilde{\boldsymbol{e}}) \quad \forall \boldsymbol{v} \in \boldsymbol{H}_{0}(\operatorname{curl}) .
$$

Altogether, we see that $\widetilde{\boldsymbol{e}} \in \boldsymbol{H}_{0}(\mathbf{c u r l})$ satisfies

$$
a(\widetilde{\boldsymbol{e}}, \boldsymbol{v}-\widetilde{\boldsymbol{e}})+\Psi(\boldsymbol{v})-\Psi(\widetilde{\boldsymbol{e}}) \geq(\mathbf{f}+\operatorname{curl} g, \boldsymbol{v}-\widetilde{\boldsymbol{e}})_{\boldsymbol{L}^{2}(\Omega)} \quad \forall \boldsymbol{v} \in \boldsymbol{H}_{0}(\operatorname{curl}) .
$$

But, we know that $\boldsymbol{e} \in \boldsymbol{H}_{0}(\mathbf{c u r l})$ is the unique solution of the above variational inequality, and hence $\widetilde{\boldsymbol{e}}=\boldsymbol{e}$. Employing this identity and (3.17) yields $\widetilde{\boldsymbol{h}}=\boldsymbol{h}$. Then, the identities $\widetilde{\boldsymbol{e}}=\boldsymbol{e}$ and $\widetilde{\boldsymbol{h}}=\boldsymbol{h}$ imply $\widetilde{\boldsymbol{j}}=\boldsymbol{j}$. This completes the proof.

Theorem 3.2. Let Assumption 2.1 be satisfied. Then, for every $y \in L^{\infty}(\Omega)$ and $(\mathbf{f}, \mathbf{g}) \in \boldsymbol{L}^{2}(\Omega) \times \boldsymbol{X}(\Omega)$, the nonlinear PDE-system

$$
\begin{cases}\epsilon \boldsymbol{e}-\operatorname{curl} \boldsymbol{h}+\boldsymbol{j}=\mathbf{f} & \text { in } \Omega \\ \mu \boldsymbol{h}+\operatorname{curl} \boldsymbol{e}=\mu \mathrm{g} & \text { in } \Omega \\ \boldsymbol{e} \times \boldsymbol{n}=0 & \text { on } \partial \Omega \\ |\boldsymbol{j}(x)| \leq j_{c}(x, y(x), \boldsymbol{h}(x)) & \text { for a.e. } x \in \Omega \\ \boldsymbol{j}(x) \cdot \boldsymbol{e}(x)=j_{c}(x, y(x), \boldsymbol{h}(x))|\boldsymbol{e}(x)| & \text { for a.e. } x \in \Omega\end{cases}
$$

admits a solution $(\boldsymbol{e}, \boldsymbol{h}, \boldsymbol{j}) \in \boldsymbol{H}_{0}(\mathbf{c u r l}) \times \boldsymbol{X}(\Omega) \times \boldsymbol{L}^{\infty}(\Omega)$. Under the smallness condition $L\left(\|y\|_{L^{\infty}(\Omega)}\right)<2 \sqrt{\underline{\epsilon} \underline{\mu}}$, the solution of (3.20) is unique.

Proof. Let $y \in L^{\infty}(\Omega)$ and $(\mathbf{f}, \mathbf{g}) \in \boldsymbol{L}^{2}(\Omega) \times \boldsymbol{X}(\Omega)$. We introduce the operator

$$
F: \boldsymbol{L}^{2}(\Omega) \rightarrow \boldsymbol{L}^{2}(\Omega), \quad \boldsymbol{z} \mapsto \boldsymbol{h},
$$

that assigns to every function $\boldsymbol{z} \in \boldsymbol{L}^{2}(\Omega)$ the unique solution $\boldsymbol{h} \in \boldsymbol{X}(\Omega)$ of the complementarity-type problem (3.1). Let us prove that $F$ is Lipschitz continuous. To this aim, let $\boldsymbol{z}_{1}, \boldsymbol{z}_{2} \in \boldsymbol{L}^{2}(\Omega)$, and let $\left(\boldsymbol{e}_{i}, \boldsymbol{h}_{i}, \boldsymbol{j}_{i}\right) \in$ 
$\boldsymbol{H}_{0}($ curl $) \times \boldsymbol{X}(\Omega) \times \boldsymbol{L}^{\infty}(\Omega)$ denote the unique solutions of (3.1) associated with $\boldsymbol{z}=\boldsymbol{z}_{i}$ for $i=1,2$. By definition,

$$
\begin{array}{r}
\epsilon\left(\boldsymbol{e}_{1}-\boldsymbol{e}_{2}\right)-\operatorname{curl}\left(\boldsymbol{h}_{1}-\boldsymbol{h}_{2}\right)+\boldsymbol{j}_{1}-\boldsymbol{j}_{2}=0 \\
\mu\left(\boldsymbol{h}_{1}-\boldsymbol{h}_{2}\right)+\operatorname{curl}\left(\boldsymbol{e}_{1}-\boldsymbol{e}_{2}\right)=0
\end{array}
$$

from which it follows that

$$
\left(\mu^{-1} \operatorname{curl}\left(e_{1}-e_{2}\right), \operatorname{curl}\left(e_{1}-e_{2}\right)\right)_{L^{2}(\Omega)}+\left(\epsilon\left(e_{1}-e_{2}\right),\left(e_{1}-e_{2}\right)\right)_{L^{2}(\Omega)}=\left(j_{2}-j_{1}, e_{1}-e_{2}\right)_{L^{2}(\Omega)} .
$$

On the other hand, according to (3.1), the right-hand side of the above identity satisfies

$$
\begin{aligned}
\left(\boldsymbol{j}_{2}-\right. & \left.\boldsymbol{j}_{1}, \boldsymbol{e}_{1}-\boldsymbol{e}_{2}\right)_{\boldsymbol{L}^{2}(\Omega)} \\
& =\int_{\Omega} \boldsymbol{j}_{2}(x) \cdot \boldsymbol{e}_{1}(x)-\boldsymbol{j}_{2}(x) \cdot \boldsymbol{e}_{2}(x)-\boldsymbol{j}_{1}(x) \cdot \boldsymbol{e}_{1}(x)+\boldsymbol{j}_{1}(x) \cdot \boldsymbol{e}_{2}(x) \mathrm{d} x \\
& =\int_{\Omega} \boldsymbol{j}_{2}(x) \cdot \boldsymbol{e}_{1}(x)-j_{c}\left(x, y(x), \boldsymbol{z}_{2}(x)\right)\left|\boldsymbol{e}_{2}(x)\right|-j_{c}\left(x, y(x), \boldsymbol{z}_{1}(x)\right)\left|\boldsymbol{e}_{1}(x)\right|+\boldsymbol{j}_{1}(x) \cdot \boldsymbol{e}_{2}(x) \mathrm{d} x \\
& \leq \int_{\Omega} j_{c}\left(x, y(x), \boldsymbol{z}_{2}(x)\right)\left(\left|\boldsymbol{e}_{1}(x)\right|-\left|\boldsymbol{e}_{2}(x)\right|\right)-j_{c}\left(x, y(x), \boldsymbol{z}_{1}(x)\right)\left(\left|\boldsymbol{e}_{1}(x)\right|-\left|\boldsymbol{e}_{2}(x)\right|\right) \mathrm{d} x \\
& =\int_{\Omega} j_{c}\left(x, y(x), \boldsymbol{z}_{2}(x)\right)-j_{c}\left(x, y(x), \boldsymbol{z}_{1}(x)\right)\left(\left|\boldsymbol{e}_{1}(x)\right|-\left|\boldsymbol{e}_{2}(x)\right|\right) \mathrm{d} x \\
& \leq \int_{\Omega} L\left(\|y\|_{L^{\infty}(\Omega)}\right)\left|\boldsymbol{z}_{2}(x)-\boldsymbol{z}_{1}(x) \| \boldsymbol{e}_{1}(x)-\boldsymbol{e}_{2}(x)\right| \mathrm{d} x \\
& \leq \frac{L\left(\|y\|_{L^{\infty}(\Omega)}\right)^{2}}{4 \underline{\epsilon}}\left\|\boldsymbol{z}_{1}-\boldsymbol{z}_{2}\right\|_{\boldsymbol{L}^{2}(\Omega)}^{2}+\left\|\epsilon^{\frac{1}{2}}\left(\boldsymbol{e}_{1}-\boldsymbol{e}_{2}\right)\right\|_{\boldsymbol{L}^{2}(\Omega)}^{2} .
\end{aligned}
$$

Applying the above inequality to (3.23) yields

$$
\left\|\mu^{\frac{1}{2}}\left(\boldsymbol{h}_{1}-\boldsymbol{h}_{2}\right)\right\|_{\boldsymbol{L}^{2}(\Omega)}^{2} \underbrace{=}_{(3.22)}\left\|\mu^{-\frac{1}{2}} \operatorname{curl}\left(\boldsymbol{e}_{1}-\boldsymbol{e}_{2}\right)\right\|_{\boldsymbol{L}^{2}(\Omega)}^{2} \leq \frac{L\left(\|y\|_{L^{\infty}(\Omega)}\right)^{2}}{4 \underline{\epsilon}}\left\|\boldsymbol{z}_{1}-\boldsymbol{z}_{2}\right\|_{\boldsymbol{L}^{2}(\Omega)}^{2},
$$

from which it follows that

$$
\left\|F\left(\boldsymbol{z}_{1}\right)-F\left(\boldsymbol{z}_{2}\right)\right\|_{\boldsymbol{L}^{2}(\Omega)} \leq \frac{L\left(\|y\|_{L^{\infty}(\Omega)}\right)}{2 \sqrt{\underline{\epsilon} \underline{\mu}}}\left\|\boldsymbol{z}_{1}-\boldsymbol{z}_{2}\right\|_{\boldsymbol{L}^{2}(\Omega)} \quad \forall \boldsymbol{z}_{1}, \boldsymbol{z}_{2} \in \boldsymbol{L}^{2}(\Omega) .
$$

Let $i$ denote the embedding $\boldsymbol{X}(\Omega) \hookrightarrow \boldsymbol{L}^{2}(\Omega)$ and

$$
\begin{aligned}
\mathbb{K}:= & \left\{\boldsymbol{q} \in \boldsymbol{X}(\Omega) \mid\|\boldsymbol{q}\|_{\boldsymbol{X}(\Omega)} \leq \bar{\epsilon}^{\frac{1}{2}}\|\mathbf{f}\|_{L_{\frac{1}{\epsilon}}^{2}(\Omega)}+\underline{\mu}^{-\frac{1}{2}}\|\mathbf{g}\|_{\boldsymbol{L}_{\mu}^{2}(\Omega)}+\left(\underline{\mu}^{-\frac{1}{2}}+\bar{\epsilon}^{\frac{1}{2}}\right)\|\mathbf{f}+\mathbf{c u r l} \mathbf{g}\|_{\boldsymbol{L}_{\frac{1}{\epsilon}}^{2}(\Omega)}\right. \\
& \left.+\sqrt{\frac{\bar{\epsilon}}{\underline{\epsilon}}} C\left(\|y\|_{L^{\infty}(\Omega)}\right)|\Omega|^{\frac{1}{2}}\right\} .
\end{aligned}
$$

By $\mathcal{K}:=\operatorname{cl}(\mathbb{K})$, we denote the closure of $\mathbb{K}$ with respect to the $\boldsymbol{L}^{2}(\Omega)$-topology. Obviously, $\mathcal{K}$ is nonempty and convex. Moreover, as the embedding $i: \boldsymbol{X}(\Omega) \hookrightarrow \boldsymbol{L}^{2}(\Omega)$ is compact $[19,26]$ and $\mathbb{K} \subset \boldsymbol{X}(\Omega)$ is bounded, the set $\mathcal{K}=\operatorname{cl}(\mathbb{K}) \subset \boldsymbol{L}^{2}(\Omega)$ is compact. Also, thanks to the a priori estimate (3.2) and the previously proven (Lipschitz) continuity result (3.24), the operator $F$ is continuous from $\mathcal{K}$ to itself, i.e., $F: \mathcal{K} \rightarrow \mathcal{K}$. In conclusion, the Schauder fixed-point theorem is applicable and guarantees the existence of a fixed point $\boldsymbol{h} \in \mathcal{K}$, i.e., $F(\boldsymbol{h})=\boldsymbol{h}$. Thus, in view of the definition of the operator $F$, the PDE-system (3.20) admits a solution $(\boldsymbol{e}, \boldsymbol{h}, \boldsymbol{j}) \in$ $\boldsymbol{H}_{0}(\mathbf{c u r l}) \times \boldsymbol{X}(\Omega) \times \boldsymbol{L}^{\infty}(\Omega)$. Finally, if $L\left(\|y\|_{L^{\infty}(\Omega)}\right)<2 \sqrt{\underline{\underline{\epsilon}} \underline{\mu}}$, then the uniqueness of the solution follows from (3.24). 
Let us close this section by presenting the variational formulation of (3.20) in terms of $\boldsymbol{e}$ :

Corollary 3.3. Let Assumption 2.1 be satisfied. Then, for every $y \in L^{\infty}(\Omega)$ and $(\mathbf{f}, \mathbf{g}) \in \boldsymbol{L}^{2}(\Omega) \times \boldsymbol{X}(\Omega)$, the $\boldsymbol{H}$ (curl)-elliptic quasi-variational inequality

$$
\begin{array}{r}
a(\boldsymbol{e}, \boldsymbol{v}-\boldsymbol{e})+\int_{\Omega} j_{c}\left(x, y(x), \mathbf{g}(x)-\mu^{-1}(x) \operatorname{curl} \boldsymbol{e}(x)\right)(|\boldsymbol{v}(x)|-|\boldsymbol{e}(x)|) \mathrm{d} x \\
\geq(\mathbf{f}+\operatorname{curl} \mathbf{g}, \boldsymbol{v}-\boldsymbol{e})_{\boldsymbol{L}^{2}(\Omega)} \quad \forall \boldsymbol{v} \in \boldsymbol{H}_{0}(\mathbf{c u r l})
\end{array}
$$

admits a solution $\boldsymbol{e} \in \boldsymbol{H}_{0}$ (curl). Furthermore, $\boldsymbol{e} \in \boldsymbol{H}_{0}(\mathbf{c u r l})$ is a solution to (EQVI) if and only if it is a solution to (3.20). Thus, if $L\left(\|y\|_{L^{\infty}(\Omega)}\right)<2 \sqrt{\underline{\epsilon} \underline{\mu}}$, the solution of (EQVI) is unique.

Proof. Let $y \in L^{\infty}(\Omega)$ and $(\mathbf{f}, \mathbf{g}) \in \boldsymbol{L}^{2}(\Omega) \times \boldsymbol{X}(\Omega)$. According to Theorem 3.2, the PDE-system (3.20) admits a solution $(\boldsymbol{e}, \boldsymbol{h}, \boldsymbol{j}) \in \boldsymbol{H}_{0}(\mathbf{c u r l}) \times \boldsymbol{X}(\Omega) \times \boldsymbol{L}^{\infty}(\Omega)$. The equalities $\epsilon \boldsymbol{e}-\operatorname{curl} \boldsymbol{h}+\boldsymbol{j}=\mathbf{f}$ and $\boldsymbol{h}=\mathbf{g}-\mu^{-1} \mathbf{c u r l} \boldsymbol{e}$ as well as (2.1) imply

$$
a(\boldsymbol{e}, \boldsymbol{v}-\boldsymbol{e})+(\boldsymbol{j}, \boldsymbol{v}-\boldsymbol{e})_{\boldsymbol{L}^{2}(\Omega)}=(\mathbf{f}+\operatorname{curl} \mathbf{g}, \boldsymbol{v}-\boldsymbol{e})_{\boldsymbol{L}^{2}(\Omega)} \quad \forall \boldsymbol{v} \in \boldsymbol{H}_{0}(\operatorname{curl}) .
$$

On the other hand, the conditions for $\boldsymbol{j}$ in (3.20) yield

$$
(\boldsymbol{j}, \boldsymbol{v}-\boldsymbol{e})_{\boldsymbol{L}^{2}(\Omega)} \leq \int_{\Omega} j_{c}(x, y(x), \boldsymbol{h}(x))|\boldsymbol{v}(x)|-j_{c}(x, y(x), \boldsymbol{h}(x))|\boldsymbol{e}(x)| \mathrm{d} x
$$

Inserting $\boldsymbol{h}=\mathbf{g}-\mu^{-1}$ curl $\boldsymbol{e}$ in (3.26) and applying the resulting inequality to (3.25), we see that $\boldsymbol{e} \in \boldsymbol{H}_{0}$ (curl) is a solution to (EQVI).

Now, suppose that $\boldsymbol{e} \in \boldsymbol{H}_{0}(\mathbf{c u r l})$ is a solution to (EQVI), i.e., it satisfies

$$
a(\boldsymbol{e}, \boldsymbol{v}-\boldsymbol{e})+\int_{\Omega} j_{c}(x, y(x), \boldsymbol{h}(x))(|\boldsymbol{v}(x)|-|\boldsymbol{e}(x)|) \mathrm{d} x \geq(\mathbf{f}+\mathbf{c u r l} \mathbf{g}, \boldsymbol{v}-\boldsymbol{e})_{\boldsymbol{L}^{2}(\Omega)} \forall \boldsymbol{v} \in \boldsymbol{H}_{0}(\mathbf{c u r l})
$$

with $\boldsymbol{h}:=\mathrm{g}-\mu^{-1}$ curl $\boldsymbol{e}$. The above problem coincides with (3.4) for $\boldsymbol{z}=\boldsymbol{h}$. Therefore, according to the proof of Lemma 3.1, there exists a $\boldsymbol{j} \in \boldsymbol{L}^{\infty}(\Omega)$ such that

$$
\begin{cases}\epsilon \boldsymbol{e}-\mathbf{c u r l} \boldsymbol{h}+\boldsymbol{j}=\mathbf{f} & \\ \boldsymbol{h}=\mathbf{g}-\mu^{-1} \mathbf{c u r l} \boldsymbol{e} \in \boldsymbol{X}(\Omega) & \text { for a.e. } x \in \Omega \\ |\boldsymbol{j}(x)| \leq j_{c}(x, y(x), \boldsymbol{h}(x)) & \text { for a.e. } x \in \Omega \\ \boldsymbol{j}(x) \cdot \boldsymbol{e}(x)=j_{c}(x, y(x), \boldsymbol{h}(x))|\boldsymbol{e}(x)|\end{cases}
$$

In conclusion, the triple $(\boldsymbol{e}, \boldsymbol{h}, \boldsymbol{j}) \in \boldsymbol{H}_{0}(\mathbf{c u r l}) \times \boldsymbol{X}(\Omega) \times \boldsymbol{L}^{\infty}(\Omega)$ is a solution to (3.20).

\section{Time-DiscRETE PROBLEM}

This section is devoted to the analysis of the time-discrete problem associated with (1.1) on the basis of the implicit Euler scheme. Let us begin by stating the required regularity assumption for the applied current source $\boldsymbol{u}: \Omega \times[0, T] \rightarrow \mathbb{R}^{3}$, the temperature distribution $\theta: \Omega \times[0, T] \rightarrow \mathbb{R}$, and the initial data $\boldsymbol{E}_{0}: \Omega \rightarrow \mathbb{R}^{3}$ and $\boldsymbol{H}_{0}: \Omega \rightarrow \mathbb{R}^{3}$.

Assumption 4.1. Suppose that

$$
\begin{aligned}
\boldsymbol{u} & \in H^{1}\left((0, T), \boldsymbol{L}^{2}(\Omega)\right) \hookrightarrow \mathcal{C}\left([0, T], \boldsymbol{L}^{2}(\Omega)\right) \\
\theta & \in H^{1}\left((0, T), L^{2}(\Omega)\right) \cap \mathcal{C}\left([0, T], L^{\infty}(\Omega)\right) \\
\left(\boldsymbol{E}_{0}, \boldsymbol{H}_{0}\right) & \in \boldsymbol{H}_{0}(\mathbf{c u r l}) \times \boldsymbol{X}(\Omega) .
\end{aligned}
$$

In Assumption 4.1 and all what follows, we use the abbreviation $\boldsymbol{u}(t)=\boldsymbol{u}(\cdot, t)$. This notation is also used for other functions acting in $\Omega \times(0, T)$. 
Remark 4.2. In real applications, the temperature distribution $\theta$ is specified by the solution of heat equations. For instance,

$$
\begin{cases}\partial_{t} \theta-\Delta \theta=f & \text { in } \Omega \times(0, T) \\ \nabla \theta \cdot \boldsymbol{n}=0 & \text { on } \partial \Omega \times(0, T) \\ \theta(0)=\theta_{0} & \text { in } \Omega\end{cases}
$$

If $\theta_{0} \in D(\Delta):=\left\{y \in H^{2}(\Omega) \mid \nabla y \cdot \boldsymbol{n}=0\right.$ on $\left.\partial \Omega\right\}, f \in W^{1,1}\left((0, T), L^{2}(\Omega)\right)$ or $f \in \mathcal{C}([0, T], D(\Delta))$, and $\Omega$ is of class $\mathcal{C}^{1,1}$, then the classical semigroup theory (see, e.g., [10], Cors. 7.6 and 7.8) implies that (4.1) admits a unique solution $\theta \in \mathcal{C}^{1}\left([0, T], L^{2}(\Omega)\right) \cap \mathcal{C}([0, T], D(\Delta))$. In particular, $\theta$ satisfies Assumption 4.1 .

Now, under Assumption 4.1, we introduce an auxiliary current density

$$
\boldsymbol{J}_{0}(x):= \begin{cases}\frac{\boldsymbol{E}_{0}(x)}{\left|\boldsymbol{E}_{0}(x)\right|} j_{c}\left(x, \theta(x, 0), \boldsymbol{H}_{0}(x)\right) & \text { if }\left|\boldsymbol{E}_{0}(x)\right| \neq 0 \\ 0 & \text { if }\left|\boldsymbol{E}_{0}(x)\right|=0\end{cases}
$$

and set

$$
\delta \boldsymbol{E}^{0}:=\epsilon^{-1}\left(\boldsymbol{u}(0)+\operatorname{curl} \boldsymbol{H}_{0}-\boldsymbol{J}_{0}\right), \quad \delta \boldsymbol{H}^{0}:=-\mu^{-1} \operatorname{curl} \boldsymbol{E}_{0} .
$$

Thus, in view of (4.2) and (4.3), it holds that

$$
\begin{cases}\epsilon \delta \boldsymbol{E}^{0}-\operatorname{curl} \boldsymbol{H}_{0}+\boldsymbol{J}_{0}=\boldsymbol{u}(0) & \text { in } \Omega \\ \mu \delta \boldsymbol{H}^{0}+\operatorname{curl} \boldsymbol{E}_{0}=0 & \text { in } \Omega \\ \left|\boldsymbol{J}_{0}(x)\right| \leq j_{c}\left(x, \theta(x, 0), \boldsymbol{H}_{0}(x)\right) & \text { for a.e. } x \in \Omega \\ \boldsymbol{J}_{0}(x) \cdot \boldsymbol{E}_{0}(x)=j_{c}\left(x, \theta(x, 0), \boldsymbol{H}_{0}(x)\right)\left|\boldsymbol{E}_{0}(x)\right| & \text { for a.e. } x \in \Omega .\end{cases}
$$

The property (4.4) is crucial for our subsequent analysis since it allows us to include the initial data $\left(\boldsymbol{E}_{0}, \boldsymbol{H}_{0}\right)$ in the time-discrete problem without any modification of the underlying QVI structure. Let us now formulate the time-discrete problem: For every $n \in \mathbb{N}$, we consider

$$
0=t_{0}<t_{1}<\ldots<t_{n}=T \quad \text { with } \quad t_{k}:=k \frac{T}{n} \quad \forall k \in\{0, \ldots, n\}
$$

and

$$
\begin{cases}\epsilon \delta \boldsymbol{E}^{k}-\operatorname{curl} \boldsymbol{H}^{k}+\boldsymbol{J}^{k}=\boldsymbol{u}\left(t_{k}\right) & \\ \mu \delta \boldsymbol{H}^{k}+\operatorname{curl} \boldsymbol{E}^{k}=0 & \text { for a.e. } x \in \Omega \\ \left|\boldsymbol{J}^{k}(x)\right| \leq j_{c}\left(x, \theta\left(x, t_{k}\right), \boldsymbol{H}^{k}(x)\right) & \text { for a.e. } x \in \Omega \\ \boldsymbol{J}^{k}(x) \cdot \boldsymbol{E}^{k}(x)=j_{c}\left(x, \theta\left(x, t_{k}\right), \boldsymbol{H}^{k}(x)\right)\left|\boldsymbol{E}^{k}(x)\right| & \\ \boldsymbol{E}^{0}=\boldsymbol{E}_{0}, \quad \boldsymbol{H}^{0}=\boldsymbol{H}_{0}, \quad \boldsymbol{J}^{0}=\boldsymbol{J}_{0} & \\ k=0, \ldots, n, & \end{cases}
$$

where

$$
\delta \boldsymbol{E}^{k}:=\frac{\boldsymbol{E}^{k}-\boldsymbol{E}^{k-1}}{n^{-1} T} \quad \text { and } \quad \delta \boldsymbol{H}^{k}:=\frac{\boldsymbol{H}^{k}-\boldsymbol{H}^{k-1}}{n^{-1} T} \quad \forall k \in\{1, \ldots, n\} .
$$

We note that $\left(\mathrm{P}_{n}\right)$ at the initial step $k=0$ is readily satisfied due to (4.4).

Theorem 4.3. Let Assumptions 2.1 and 4.1 be satisfied. Then, for every $n \in \mathbb{N}$, the time-discrete problem $\left(\mathrm{P}_{n}\right)$ admits a solution $\left\{\left(\boldsymbol{E}^{k}, \boldsymbol{H}^{k}, \boldsymbol{J}^{k}\right)\right\}_{k=1}^{n} \subset \boldsymbol{H}_{0}(\mathbf{c u r l}) \times \boldsymbol{X}(\Omega) \times \boldsymbol{L}^{\infty}(\Omega)$. If $n \in \mathbb{N}$ is sufficiently large, then the solution of $\left(\mathrm{P}_{n}\right)$ is unique. 
Proof. Thanks to Assumption 4.1, the existence result for $\left(\mathrm{P}_{n}\right)$ is an immediate consequence of Theorem 3.2. Thus, it remains to prove that, for all sufficiently large $n \in \mathbb{N}$, the solution of $\left(\mathrm{P}_{n}\right)$ is unique. To show this, let us notice that for every $k=1, \ldots, n,\left(\boldsymbol{E}^{k}, \boldsymbol{H}^{k}, \boldsymbol{J}^{k}\right) \in \boldsymbol{H}_{0}(\mathbf{c u r l}) \times \boldsymbol{X}(\Omega) \times \boldsymbol{L}^{\infty}(\Omega)$ is a solution to

$$
\begin{cases}\frac{\epsilon}{n^{-1} T} \boldsymbol{\mu}-\operatorname{curl} \boldsymbol{h}+\boldsymbol{j}=\mathbf{f}^{k} & \text { in } \Omega \\ \frac{\mu}{n^{-1} T} \boldsymbol{h}+\operatorname{curl} \boldsymbol{e}=\mu \mathbf{g}^{k} & \text { in } \Omega \\ \boldsymbol{e} \times \boldsymbol{n}=0 & \text { on } \partial \Omega \\ |\boldsymbol{j}(x)| \leq j_{c}\left(x, \theta\left(x, t_{k}\right), \boldsymbol{h}(x)\right) & \text { for a.e. } x \in \Omega \\ \boldsymbol{j}(x) \cdot \boldsymbol{e}(x)=j_{c}\left(x, \theta\left(x, t_{k}\right), \boldsymbol{h}(x)\right)|\boldsymbol{e}(x)| & \text { for a.e. } x \in \Omega\end{cases}
$$

with $\mathbf{f}^{k}:=\boldsymbol{u}\left(t_{k}\right)+\frac{\epsilon}{n^{-1} T} \boldsymbol{E}^{k-1} \in \boldsymbol{L}^{2}(\Omega)$ and $\mathbf{g}^{k}:=\frac{1}{n^{-1} T} \boldsymbol{H}^{k-1} \in \boldsymbol{X}(\Omega)$. Analogously to the proof of Theorem 3.2, we introduce the fixed-point mapping $F_{k}: \boldsymbol{L}^{2}(\Omega) \rightarrow \boldsymbol{L}^{2}(\Omega)$ associated with (4.6). Due to the presence of the time step $n^{-1} T$ in (4.6), we obtain as in (3.24) that the mapping $F_{k}: \boldsymbol{L}^{2}(\Omega) \rightarrow \boldsymbol{L}^{2}(\Omega)$ has the Lipschitz constant $\frac{T L\left(\|\theta\|_{\mathcal{C}([0, T], L}(\Omega)\right)}{2 n \sqrt{\underline{\underline{\epsilon}} \underline{\mu}}}$. Thus, for every $n \in \mathbb{N}$ satisfying $n>\frac{T L\left(\|\theta\|_{\mathcal{C}([0, T], L \infty(\Omega)}\right)}{2 \sqrt{\underline{\underline{\underline{\mu}}}}}, F_{k}: \boldsymbol{L}^{2}(\Omega) \rightarrow \boldsymbol{L}^{2}(\Omega)$ turns out to be a contraction. In conclusion, the Banach fixed-point theorem implies that (4.6) admits a unique solution.

In the following, we shall make use of the classical discrete Gronwall lemma. For the convenience of the reader, we recall it in the following lemma:

Lemma 4.4. Let $\left\{a_{k}\right\}_{k=0}^{\infty}$ and $\left\{b_{k}\right\}_{k=0}^{\infty}$ be sequences of nonnegative real numbers satisfying

$$
a_{m} \leq c+\sum_{k=0}^{m-1} a_{k} b_{k} \quad \forall m \in \mathbb{N}
$$

for some constant $c>0$. Then, it holds that

$$
a_{m} \leq c \exp \left(\sum_{k=0}^{m-1} b_{k}\right) \quad \forall m \in \mathbb{N} .
$$

Lemma 4.5. Let Assumptions 2.1 and 4.1 be satisfied. Furthermore, let $n \in \mathbb{N}$, and let $\left\{\left(\boldsymbol{E}^{k}, \boldsymbol{H}^{k}, \boldsymbol{J}^{k}\right)\right\}_{k=1}^{n} \subset$ $\boldsymbol{H}_{0}(\mathbf{c u r l}) \times \boldsymbol{X}(\Omega) \times \boldsymbol{L}^{\infty}(\Omega)$ denote a solution to $\left(\mathrm{P}_{n}\right)$. Then, for all $m=1, \ldots, n$, it holds that

$$
\begin{aligned}
\left\|\boldsymbol{E}^{m}\right\|_{\boldsymbol{L}_{\epsilon}^{2}(\Omega)}^{2}+ & \left\|\boldsymbol{H}^{m}\right\|_{\boldsymbol{L}_{\mu}^{2}(\Omega)}^{2}+\sum_{k=1}^{m}\left(\left\|\boldsymbol{E}^{k}-\boldsymbol{E}^{k-1}\right\|_{\boldsymbol{L}_{\epsilon}^{2}(\Omega)}^{2}+\left\|\boldsymbol{H}^{k}-\boldsymbol{H}^{k-1}\right\|_{\boldsymbol{L}_{\mu}^{2}(\Omega)}^{2}\right) \\
& \leq(e+1)\left(\left\|\boldsymbol{E}_{0}\right\|_{\boldsymbol{L}_{\epsilon}^{2}(\Omega)}^{2}+\left\|\boldsymbol{H}_{0}\right\|_{\boldsymbol{L}_{\mu}^{2}(\Omega)}^{2}+2 T^{2} \underline{\epsilon}^{-1}\|\boldsymbol{u}\|_{\mathcal{C}\left([0, T], \boldsymbol{L}^{2}(\Omega)\right)}^{2}\right) .
\end{aligned}
$$

Proof. Let $m \in\{1, \ldots, n\}$ and $k \in\{1, \ldots m\}$. The first equality of $\left(\mathrm{P}_{n}\right)$ implies

$$
\left(\epsilon \frac{\boldsymbol{E}^{k}-\boldsymbol{E}^{k-1}}{n^{-1} T}-\operatorname{curl} \boldsymbol{H}^{k}+\boldsymbol{J}^{k}, \boldsymbol{E}^{k}\right)_{\boldsymbol{L}^{2}(\Omega)}=\left(\boldsymbol{u}\left(t_{k}\right), \boldsymbol{E}^{k}\right)_{\boldsymbol{L}^{2}(\Omega)} .
$$

On the other hand, the second equality of $\left(\mathrm{P}_{n}\right)$ yields

$$
\left(\operatorname{curl} \boldsymbol{H}^{k}, \boldsymbol{E}^{k}\right)_{\boldsymbol{L}^{2}(\Omega)} \underbrace{=}_{(2.1)}\left(\boldsymbol{H}^{k}, \operatorname{curl} \boldsymbol{E}^{k}\right)_{\boldsymbol{L}^{2}(\Omega)}=-\left(\boldsymbol{H}^{k}, \frac{\boldsymbol{H}^{k}-\boldsymbol{H}^{k-1}}{n^{-1} T}\right)_{\boldsymbol{L}_{\mu}^{2}(\Omega)} .
$$


Applying the inequality $\boldsymbol{J}^{k}(x) \cdot \boldsymbol{E}^{k}(x)=j_{c}\left(x, \theta\left(x, t_{k}\right), \boldsymbol{H}^{k}(x)\right)\left|\boldsymbol{E}^{k}(x)\right| \geq 0$ for a.e. $x \in \Omega$ and (4.9) to (4.8), we obtain that

$$
\sum_{k=1}^{m}\left(\left(\boldsymbol{E}^{k}-\boldsymbol{E}^{k-1}, \boldsymbol{E}^{k}\right)_{\boldsymbol{L}_{\epsilon}^{2}(\Omega)}+\left(\boldsymbol{H}^{k}-\boldsymbol{H}^{k-1}, \boldsymbol{H}^{k}\right)_{\boldsymbol{L}_{\mu}^{2}(\Omega)}\right) \leq \sum_{k=1}^{m} \frac{T}{n}\left(\boldsymbol{u}\left(t_{k}\right), \boldsymbol{E}^{k}\right)_{\boldsymbol{L}^{2}(\Omega)} .
$$

The first sum involving the electric field can be written as follows:

$$
\begin{aligned}
\sum_{k=1}^{m}\left(\boldsymbol{E}^{k}-\boldsymbol{E}^{k-1}, \boldsymbol{E}^{k}\right)_{\boldsymbol{L}_{\epsilon}^{2}(\Omega)} & =\sum_{k=1}^{m}\left\|\boldsymbol{E}^{k}-\boldsymbol{E}^{k-1}\right\|_{\boldsymbol{L}_{\epsilon}^{2}(\Omega)}^{2}+\sum_{k=1}^{m}\left(\boldsymbol{E}^{k}-\boldsymbol{E}^{k-1}, \boldsymbol{E}^{k-1}\right)_{\boldsymbol{L}_{\epsilon}^{2}(\Omega)} \\
& =\sum_{k=1}^{m}\left\|\boldsymbol{E}^{k}-\boldsymbol{E}^{k-1}\right\|_{\boldsymbol{L}_{\epsilon}^{2}(\Omega)}^{2}+\left\|\boldsymbol{E}^{m}\right\|_{\boldsymbol{L}_{\epsilon}^{2}(\Omega)}^{2}-\left\|\boldsymbol{E}_{0}\right\|_{\boldsymbol{L}_{\epsilon}^{2}(\Omega)}^{2}-\sum_{k=1}^{m}\left(\boldsymbol{E}^{k}-\boldsymbol{E}^{k-1}, \boldsymbol{E}^{k}\right)_{\boldsymbol{L}_{\epsilon}^{2}(\Omega)}
\end{aligned}
$$

where we have used the summation by parts formula. A similar result holds also for the second sum in (4.10). Altogether, we obtain

$$
\begin{gathered}
\left\|\boldsymbol{E}^{m}\right\|_{\boldsymbol{L}_{\epsilon}^{2}(\Omega)}^{2}+\left\|\boldsymbol{H}^{m}\right\|_{\boldsymbol{L}_{\mu}^{2}(\Omega)}^{2}+\sum_{k=1}^{m}\left(\left\|\boldsymbol{E}^{k}-\boldsymbol{E}^{k-1}\right\|_{\boldsymbol{L}_{\epsilon}^{2}(\Omega)}^{2}+\left\|\boldsymbol{H}^{k}-\boldsymbol{H}^{k-1}\right\|_{\boldsymbol{L}_{\mu}^{2}(\Omega)}^{2}\right) \\
\leq\left\|\boldsymbol{E}_{0}\right\|_{\boldsymbol{L}_{\epsilon}^{2}(\Omega)}^{2}+\left\|\boldsymbol{H}_{0}\right\|_{\boldsymbol{L}_{\mu}^{2}(\Omega)}^{2}+2 \sum_{k=1}^{m} \frac{T}{n} \underline{\epsilon}^{-1 / 2}\left\|\boldsymbol{u}\left(t_{k}\right)\right\|_{\boldsymbol{L}^{2}(\Omega)}\left\|\boldsymbol{E}^{k}\right\|_{\boldsymbol{L}_{\epsilon}^{2}(\Omega)} \\
\leq\left\|\boldsymbol{E}_{0}\right\|_{\boldsymbol{L}_{\epsilon}^{2}(\Omega)}^{2}+\left\|\boldsymbol{H}_{0}\right\|_{\boldsymbol{L}_{\mu}^{2}(\Omega)}^{2}+2 \sum_{k=1}^{m} \frac{T}{n}\left(T \underline{\epsilon}^{-1}\left\|\boldsymbol{u}\left(t_{k}\right)\right\|_{\boldsymbol{L}^{2}(\Omega)}^{2}+\frac{1}{4 T}\left\|\boldsymbol{E}^{k}\right\|_{\boldsymbol{L}_{\epsilon}^{2}(\Omega)}^{2}\right) \\
\leq\left\|\boldsymbol{E}_{0}\right\|_{\boldsymbol{L}_{\epsilon}^{2}(\Omega)}^{2}+\left\|\boldsymbol{H}_{0}\right\|_{\boldsymbol{L}_{\mu}^{2}(\Omega)}^{2}+2 T^{2} \underline{\epsilon}^{-1}\|\boldsymbol{u}\|_{\mathcal{C}\left([0, T], \boldsymbol{L}^{2}(\Omega)\right)}^{2}+\sum_{k=1}^{m} \frac{1}{2 n}\left\|\boldsymbol{E}^{k}\right\|_{\boldsymbol{L}_{\epsilon}^{2}(\Omega)}^{2} .
\end{gathered}
$$

In particular, the estimate (4.11) implies

$$
\frac{1}{2}\left\|\boldsymbol{E}^{m}\right\|_{\boldsymbol{L}_{\epsilon}^{2}(\Omega)}^{2} \leq\left\|\boldsymbol{E}_{0}\right\|_{\boldsymbol{L}_{\epsilon}^{2}(\Omega)}^{2}+\left\|\boldsymbol{H}_{0}\right\|_{\boldsymbol{L}_{\mu}^{2}(\Omega)}^{2}+2 T^{2} \underline{\epsilon}^{-1}\|\boldsymbol{u}\|_{\mathcal{C}\left([0, T], \boldsymbol{L}^{2}(\Omega)\right)}^{2}+\sum_{k=1}^{m-1} \frac{1}{2 n}\left\|\boldsymbol{E}^{k}\right\|_{\boldsymbol{L}_{\epsilon}^{2}(\Omega)}^{2},
$$

and consequently, by Lemma 4.4, we obtain that

$$
\frac{1}{2}\left\|\boldsymbol{E}^{k}\right\|_{\boldsymbol{L}_{\epsilon}^{2}(\Omega)}^{2} \leq\left(\left\|\boldsymbol{E}_{0}\right\|_{\boldsymbol{L}_{\epsilon}^{2}(\Omega)}^{2}+\left\|\boldsymbol{H}_{0}\right\|_{\boldsymbol{L}_{\mu}^{2}(\Omega)}^{2}+2 T^{2} \underline{\epsilon}^{-1}\|\boldsymbol{u}\|_{\mathcal{C}\left([0, T], \boldsymbol{L}^{2}(\Omega)\right)}^{2}\right) e \quad \forall k=1, \ldots, m .
$$

Applying (4.12) to (4.11), we come to the conclusion that the estimate (4.7) is valid for all $m=1, \ldots, n$.

In the following, for simplicity, we use the notation $j_{c}^{k}:=j_{c}\left(\cdot, \theta\left(t_{k}\right), \boldsymbol{H}^{k}\right)$. According to $\left(\mathrm{P}_{n}\right)$, it holds for all $k=0, \ldots, n$ that

$$
\left|\boldsymbol{J}^{k}(x)\right| \leq j_{c}^{k}(x) \quad \text { and } \quad \boldsymbol{J}^{k}(x) \cdot \boldsymbol{E}^{k}(x)=j_{c}^{k}(x)\left|\boldsymbol{E}^{k}(x)\right| \quad \text { for a.e. } x \in \Omega .
$$

Our next goal is to analyze the stability property for $\delta \boldsymbol{E}^{k}$ and $\delta \boldsymbol{H}^{k}$. In our analysis, we shall make use of the following constants:

$$
\begin{aligned}
\alpha & :=\left\|\delta \boldsymbol{E}^{0}\right\|_{\boldsymbol{L}_{\epsilon}^{2}(\Omega)}^{2}+\left\|\operatorname{curl} \boldsymbol{E}_{0}\right\|_{\boldsymbol{L}_{\frac{1}{\mu}}^{2}(\Omega)}^{2}+T \underline{\epsilon}^{-1}\left[2 L\left(\|\theta\|_{\mathcal{C}\left([0, T], L^{\infty}(\Omega)\right)}\right)^{2}\left\|\partial_{t} \theta\right\|_{L^{2}\left((0, T), L^{2}(\Omega)\right)}^{2}+\left\|\partial_{t} \boldsymbol{u}\right\|_{L^{2}\left((0, T), L^{2}(\Omega)\right)}^{2}\right] \\
\beta & :=2 \max \left\{2,2 T^{2} \underline{\mu}^{-1} \underline{\epsilon}^{-1} L\left(\|\theta\|_{\mathcal{C}\left([0, T], L^{\infty}(\Omega)\right)}\right)^{2}\right\} .
\end{aligned}
$$


Lemma 4.6. Let Assumptions 2.1 and 4.1 be satisfied. Furthermore, let $n \in \mathbb{N}$ satisfying $n \geq \beta$, and let $\left\{\left(\boldsymbol{E}^{k}, \boldsymbol{H}^{k}, \boldsymbol{J}^{k}\right)\right\}_{k=1}^{n} \subset \boldsymbol{H}_{0}(\mathbf{c u r l}) \times \boldsymbol{X}(\Omega) \times \boldsymbol{L}^{\infty}(\Omega)$ denote a solution to $\left(\mathrm{P}_{n}\right)$. Then, for all $m=1, \ldots, n$, it holds that

$$
\begin{aligned}
\frac{1}{2}\left\|\delta \boldsymbol{E}^{m}\right\|_{\boldsymbol{L}_{\epsilon}^{2}(\Omega)}^{2} & +\frac{1}{2}\left\|\operatorname{curl} \boldsymbol{E}^{m}\right\|_{\boldsymbol{L}_{\frac{1}{\mu}}^{2}(\Omega)}^{2}+\sum_{k=1}^{m}\left\|\delta \boldsymbol{E}^{k}-\delta \boldsymbol{E}^{k-1}\right\|_{\boldsymbol{L}_{\epsilon}^{2}(\Omega)}^{2} \\
& +\sum_{k=1}^{m}\left\|\operatorname{curl}\left(\boldsymbol{E}^{k}-\boldsymbol{E}^{k-1}\right)\right\|_{\boldsymbol{L}_{\frac{1}{\mu}}^{2}(\Omega)}^{2} \leq \alpha(1+\beta \exp (\beta)) \\
\left\|\delta \boldsymbol{H}^{m}\right\|_{\boldsymbol{L}_{\mu}^{2}(\Omega)} & \leq \sqrt{2 \alpha \exp (\beta)} \\
\left\|\operatorname{curl} \boldsymbol{H}^{m}\right\|_{\boldsymbol{L}^{2}(\Omega)} & \leq \sqrt{2 \bar{\epsilon} \alpha \exp (\beta)}+\|\boldsymbol{u}\|_{\mathcal{C}\left([0, T], \boldsymbol{L}^{2}(\Omega)\right)}+|\Omega|^{\frac{1}{2}} C\left(\|\theta\|_{\mathcal{C}\left([0, T], L^{\infty}(\Omega)\right)}\right) .
\end{aligned}
$$

Proof. Let $m \in\{1, \ldots, n\}$ and $k \in\{1, \ldots m\}$. By the first equality in $\left(\mathrm{P}_{n}\right)$, we obtain

$$
\begin{array}{r}
\left(\delta \boldsymbol{E}^{k}-\delta \boldsymbol{E}^{k-1}, \delta \boldsymbol{E}^{k}\right)_{\boldsymbol{L}_{\epsilon}^{2}(\Omega)}-\left(\operatorname{curl}\left(\boldsymbol{H}^{k}-\boldsymbol{H}^{k-1}\right), \delta \boldsymbol{E}^{k}\right)_{\boldsymbol{L}^{2}(\Omega)}+\left(\boldsymbol{J}^{k}-\boldsymbol{J}^{k-1}, \delta \boldsymbol{E}^{k}\right)_{\boldsymbol{L}^{2}(\Omega)} \\
=\left(\boldsymbol{u}\left(t_{k}\right)-\boldsymbol{u}\left(t_{k-1}\right), \delta \boldsymbol{E}^{k}\right)_{\boldsymbol{L}^{2}(\Omega)}
\end{array}
$$

On the other hand, from the second equality in $\left(\mathrm{P}_{n}\right)$, we know that

$$
\begin{aligned}
\left(\operatorname{curl}\left(\boldsymbol{H}^{k}-\boldsymbol{H}^{k-1}\right), \delta \boldsymbol{E}^{k}\right)_{\boldsymbol{L}^{2}(\Omega)} & =-\frac{T}{n}\left(\mu^{-1} \operatorname{curl} \boldsymbol{E}^{k}, \operatorname{curl} \delta \boldsymbol{E}^{k}\right)_{\boldsymbol{L}^{2}(\Omega)} \\
& =-\left(\operatorname{curl} \boldsymbol{E}^{k}, \operatorname{curl}\left(\boldsymbol{E}^{k}-\boldsymbol{E}^{k-1}\right)\right)_{\boldsymbol{L}_{\frac{1}{\mu}}^{2}(\Omega)}
\end{aligned}
$$

Combining the above two identities results in

$$
\begin{gathered}
\sum_{k=1}^{m}\left(\delta \boldsymbol{E}^{k}-\delta \boldsymbol{E}^{k-1}, \delta \boldsymbol{E}^{k}\right)_{\boldsymbol{L}_{\epsilon}^{2}(\Omega)}+\sum_{k=1}^{m}\left(\operatorname{curl} \boldsymbol{E}^{k}, \operatorname{curl}\left(\boldsymbol{E}^{k}-\boldsymbol{E}^{k-1}\right)\right)_{\boldsymbol{L}_{\frac{1}{\mu}}^{2}(\Omega)} \\
=\sum_{k=1}^{m}\left(\boldsymbol{J}^{k-1}-\boldsymbol{J}^{k}, \delta \boldsymbol{E}^{k}\right)_{\boldsymbol{L}^{2}(\Omega)}+\sum_{k=1}^{m}\left(\boldsymbol{u}\left(t_{k}\right)-\boldsymbol{u}\left(t_{k-1}\right), \delta \boldsymbol{E}^{k}\right)_{\boldsymbol{L}^{2}(\Omega)}
\end{gathered}
$$

Then, similarly to the previous lemma, the summation by parts formula implies

$$
\begin{aligned}
& \left\|\delta \boldsymbol{E}^{m}\right\|_{\boldsymbol{L}_{\epsilon}^{2}(\Omega)}^{2}-\left\|\delta \boldsymbol{E}^{0}\right\|_{\boldsymbol{L}_{\epsilon}^{2}(\Omega)}^{2}+\sum_{k=1}^{m}\left\|\delta \boldsymbol{E}^{k}-\delta \boldsymbol{E}^{k-1}\right\|_{\boldsymbol{L}_{\epsilon}^{2}(\Omega)}^{2} \\
& +\left\|\operatorname{curl} \boldsymbol{E}^{m}\right\|_{\boldsymbol{L}_{\frac{1}{\mu}}^{2}(\Omega)}^{2}-\left\|\operatorname{curl} \boldsymbol{E}_{0}\right\|_{\boldsymbol{L}_{\frac{1}{\mu}}^{2}(\Omega)}^{2}+\sum_{k=1}^{m}\left\|\operatorname{curl}\left(\boldsymbol{E}^{k}-\boldsymbol{E}^{k-1}\right)\right\|_{\boldsymbol{L}_{\frac{1}{\mu}}^{2}(\Omega)}^{2} \\
& =\underbrace{2 \sum_{k=1}^{m}\left(\boldsymbol{J}^{k-1}-\boldsymbol{J}^{k}, \delta \boldsymbol{E}^{k}\right)_{\boldsymbol{L}^{2}(\Omega)}}_{=: \mathrm{I}}+\underbrace{2 \sum_{k=1}^{m}\left(\boldsymbol{u}\left(t_{k}\right)-\boldsymbol{u}\left(t_{k-1}\right), \delta \boldsymbol{E}^{k}\right)_{\boldsymbol{L}^{2}(\Omega)}}_{=: \mathrm{II}} .
\end{aligned}
$$


Let us derive an appropriate upper estimate for $I$. According to (4.5) and (4.13), it holds that

$$
\begin{aligned}
\left(\boldsymbol{J}^{k-1}-\boldsymbol{J}^{k}, \delta \boldsymbol{E}^{k}\right)_{\boldsymbol{L}^{2}(\Omega)} & =\frac{n}{T} \int_{\Omega} \boldsymbol{J}^{k-1} \cdot \boldsymbol{E}^{k}-j_{c}^{k-1}\left|\boldsymbol{E}^{k-1}\right|+j^{k} \cdot \boldsymbol{E}^{k-1}-j_{c}^{k}\left|\boldsymbol{E}^{k}\right| \mathrm{d} x \\
& \leq \frac{n}{T} \int_{\Omega} j_{c}^{k-1}\left|\boldsymbol{E}^{k}\right|-j_{c}^{k-1}\left|\boldsymbol{E}^{k-1}\right|+j_{c}^{k}\left|\boldsymbol{E}^{k-1}\right|-j_{c}^{k}\left|\boldsymbol{E}^{k}\right| \mathrm{d} x \\
& =\frac{n}{T} \int_{\Omega}\left(j_{c}^{k-1}-j_{c}^{k}\right)\left(\left|\boldsymbol{E}^{k}\right|-\left|\boldsymbol{E}^{k-1}\right|\right) \mathrm{d} x \\
& \leq \int_{\Omega}\left|j_{c}^{k-1}-j_{c}^{k}\right|\left|\delta \boldsymbol{E}^{k}\right| \mathrm{d} x .
\end{aligned}
$$

Then, as a consequence of (A4), the second equality of $\left(\mathrm{P}_{n}\right)$, and Young's inequality, we obtain

$$
\begin{aligned}
\left(\boldsymbol{J}^{k-1}-\boldsymbol{J}^{k}, \delta \boldsymbol{E}^{k}\right)_{\boldsymbol{L}^{2}(\Omega)} \\
\leq \leq \underline{\epsilon}^{-\frac{1}{2}}\left\|j_{c}^{k-1}-j_{c}^{k}\right\|_{L^{2}(\Omega)}\left\|\delta \boldsymbol{E}^{k}\right\|_{\boldsymbol{L}_{\epsilon}^{2}(\Omega)} \\
\leq \leq \underline{\epsilon}^{-\frac{1}{2}} L\left(\|\theta\|_{\mathcal{C}\left([0, T], L^{\infty}(\Omega)\right)}\right)\left(\int_{\Omega}\left(\left|\theta\left(x, t_{k-1}\right)-\theta\left(x, t_{k}\right)\right|+\left|\boldsymbol{H}^{k-1}(x)-\boldsymbol{H}^{k}(x)\right|\right)^{2} \mathrm{~d} x\right)^{1 / 2}\left\|\delta \boldsymbol{E}^{k}\right\|_{\boldsymbol{L}_{\epsilon}^{2}(\Omega)} \\
\leq \leq \underline{\epsilon}^{-1} L\left(\|\theta\|_{\mathcal{C}\left([0, T], L^{\infty}(\Omega)\right)}\right)^{2} n\left\|\theta\left(t_{k}\right)-\theta\left(t_{k-1}\right)\right\|_{L^{2}(\Omega)}^{2}+\frac{1}{4 n}\left\|\delta \boldsymbol{E}^{k}\right\|_{\boldsymbol{L}_{\epsilon}^{2}(\Omega)}^{2} \\
\quad+\underline{\epsilon}^{-1} L\left(\|\theta\|_{\mathcal{C}\left([0, T], L^{\infty}(\Omega)\right)}\right)^{2} \frac{T^{2}}{n}\left\|\delta \boldsymbol{H}^{k}\right\|_{\boldsymbol{L}^{2}(\Omega)}^{2}+\frac{1}{4 n}\left\|\delta \boldsymbol{E}^{k}\right\|_{\boldsymbol{L}_{\epsilon}^{2}(\Omega)}^{2} \\
\leq \leq \underline{\epsilon}^{-1} L\left(\|\theta\|_{\mathcal{C}\left([0, T], L^{\infty}(\Omega)\right)}\right)^{2}\left(n\left\|\theta\left(t_{k}\right)-\theta\left(t_{k-1}\right)\right\|_{L^{2}(\Omega)}^{2}+\frac{T^{2}}{n \mu}\left\|\mathbf{c u r l} \boldsymbol{E}^{k}\right\|_{\boldsymbol{L}_{\frac{1}{\mu}}^{2}(\Omega)}^{2}\right)+\frac{1}{2 n}\left\|\delta \boldsymbol{E}^{k}\right\|_{\boldsymbol{L}_{\epsilon}^{2}(\Omega)}^{2}
\end{aligned}
$$

Since $\theta \in H^{1}\left((0, T), L^{2}(\Omega)\right)$, it holds for all $k=1, \ldots, n$ that

$$
\begin{aligned}
\left\|\theta\left(t_{k}\right)-\theta\left(t_{k-1}\right)\right\|_{L^{2}(\Omega)} & \leq \int_{t_{k-1}}^{t_{k}}\left\|\partial_{t} \theta(t)\right\|_{L^{2}(\Omega)} \mathrm{d} t \\
& \leq\left(\int_{t_{k-1}}^{t_{k}}\left\|\partial_{t} \theta(t)\right\|_{\boldsymbol{L}^{2}(\Omega)}^{2} \mathrm{~d} t\right)^{\frac{1}{2}}\left(\int_{t_{k-1}}^{t_{k}} 1 \mathrm{~d} t\right)^{\frac{1}{2}}=\sqrt{\frac{T}{n}}\left(\int_{t_{k-1}}^{t_{k}}\left\|\partial_{t} \theta(t)\right\|_{\boldsymbol{L}^{2}(\Omega)}^{2} \mathrm{~d} t\right)^{\frac{1}{2}} .
\end{aligned}
$$

Therefore,

$$
\sum_{k=1}^{m} n\left\|\theta\left(t_{k}\right)-\theta\left(t_{k-1}\right)\right\|_{L^{2}(\Omega)}^{2} \leq T \sum_{k=1}^{n} \int_{t_{k-1}}^{t_{k}}\left\|\partial_{t} \theta(t)\right\|_{L^{2}(\Omega)}^{2} \mathrm{~d} t=T\left\|\partial_{t} \theta\right\|_{L^{2}\left((0, T), L^{2}(\Omega)\right)}^{2} .
$$

Collecting all above estimates, we arrive at

$$
\begin{aligned}
\mathrm{I}=2 \sum_{k=1}^{m}\left(\boldsymbol{J}^{k-1}-\boldsymbol{J}^{k}, \delta \boldsymbol{E}^{k}\right)_{\boldsymbol{L}^{2}(\Omega)} \leq & \frac{T}{\underline{\epsilon}} 2 L\left(\|\theta\|_{\mathcal{C}\left([0, T], L^{\infty}(\Omega)\right)}\right)^{2}\left(\left\|\partial_{t} \theta\right\|_{L^{2}\left((0, T), L^{2}(\Omega)\right)}^{2}\right. \\
& \left.+\frac{T}{\underline{\mu}} \sum_{k=1}^{m} \frac{1}{n}\left\|\operatorname{curl} \boldsymbol{E}^{k}\right\|_{\boldsymbol{L}_{\frac{1}{\mu}}^{2}(\Omega)}^{2}\right)+\sum_{k=1}^{m} \frac{1}{n}\left\|\delta \boldsymbol{E}^{k}\right\|_{\boldsymbol{L}_{\epsilon}^{2}(\Omega)}^{2}
\end{aligned}
$$


Similarly, by virtue of $\boldsymbol{u} \in H^{1}\left((0, T), \boldsymbol{L}^{2}(\Omega)\right)$, we deduce that

$$
\begin{aligned}
\mathrm{II} & =2 \sum_{k=1}^{m}\left(\boldsymbol{u}\left(t_{k}\right)-\boldsymbol{u}\left(t_{k-1}\right), \delta \boldsymbol{E}^{k}\right)_{\boldsymbol{L}^{2}(\Omega)} \leq 2 \sum_{k=1}^{m} \underline{\epsilon}^{-\frac{1}{2}}\left\|\boldsymbol{u}\left(t_{k}\right)-\boldsymbol{u}\left(t_{k-1}\right)\right\|_{\boldsymbol{L}^{2}(\Omega)}\left\|\delta \boldsymbol{E}^{k}\right\|_{\boldsymbol{L}_{\epsilon}^{2}(\Omega)} \\
& \leq \sum_{k=1}^{m} \underline{\epsilon}^{-1} n\left\|\boldsymbol{u}\left(t_{k}\right)-\boldsymbol{u}\left(t_{k-1}\right)\right\|_{\boldsymbol{L}^{2}(\Omega)}^{2}+\sum_{k=1}^{m} \frac{1}{n}\left\|\delta \boldsymbol{E}^{k}\right\|_{\boldsymbol{L}_{\epsilon}^{2}(\Omega)}^{2} \\
& \leq \frac{T}{\underline{\epsilon}}\left\|\partial_{t} \boldsymbol{u}\right\|_{L^{2}\left((0, T), \boldsymbol{L}^{2}(\Omega)\right)}^{2}+\sum_{k=1}^{m} \frac{1}{n}\left\|\delta \boldsymbol{E}^{k}\right\|_{\boldsymbol{L}_{\epsilon}^{2}(\Omega)}^{2}
\end{aligned}
$$

Now, applying (4.17) and (4.18) to (4.16) yields

$$
\begin{aligned}
\left\|\delta \boldsymbol{E}^{m}\right\|_{\boldsymbol{L}_{\epsilon}^{2}(\Omega)}^{2} & +\left\|\operatorname{curl} \boldsymbol{E}^{m}\right\|_{\boldsymbol{L}_{\frac{1}{\mu}}^{2}(\Omega)}^{2}+\sum_{k=1}^{m}\left(\left\|\delta \boldsymbol{E}^{k}-\delta \boldsymbol{E}^{k-1}\right\|_{\boldsymbol{L}_{\epsilon}^{2}(\Omega)}^{2}+\left\|\operatorname{curl}\left(\boldsymbol{E}^{k}-\boldsymbol{E}^{k-1}\right)\right\|_{\boldsymbol{L}_{\frac{1}{\mu}}^{2}(\Omega)}^{2}\right) \\
& \leq \alpha+\sum_{k=1}^{m} \frac{\beta}{n}\left(\frac{1}{2}\left\|\delta \boldsymbol{E}^{k}\right\|_{\boldsymbol{L}_{\epsilon}^{2}(\Omega)}^{2}+\frac{1}{2}\left\|\operatorname{curl} \boldsymbol{E}^{k}\right\|_{\boldsymbol{L}_{\frac{1}{\mu}}^{2}(\Omega)}^{2}\right)
\end{aligned}
$$

with $\alpha, \beta$ as in (4.14). Since $n \geq \beta$, (4.19) implies

$$
\frac{1}{2}\left\|\delta \boldsymbol{E}^{m}\right\|_{\boldsymbol{L}_{\epsilon}^{2}(\Omega)}^{2}+\frac{1}{2}\left\|\operatorname{curl} \boldsymbol{E}^{m}\right\|_{\boldsymbol{L}_{\frac{1}{\mu}}^{2}(\Omega)}^{2} \leq \alpha+\sum_{k=1}^{m-1} \frac{\beta}{n}\left(\frac{1}{2}\left\|\delta \boldsymbol{E}^{k}\right\|_{\boldsymbol{L}_{\epsilon}^{2}(\Omega)}^{2}+\frac{1}{2}\left\|\operatorname{curl} \boldsymbol{E}^{k}\right\|_{\boldsymbol{L}_{\frac{1}{\mu}}^{2}(\Omega)}^{2}\right),
$$

and so, by the discrete Gronwall lemma (Lem. 4.4),

$$
\frac{1}{2}\left\|\delta \boldsymbol{E}^{k}\right\|_{\boldsymbol{L}_{\epsilon}^{2}(\Omega)}^{2}+\frac{1}{2}\left\|\operatorname{curl} \boldsymbol{E}^{k}\right\|_{\boldsymbol{L}_{\frac{1}{\mu}}^{2}(\Omega)}^{2} \leq \alpha \exp (\beta) \quad \forall k=1, \ldots, m .
$$

Thus, the estimate (4.15a) follows immediately from (4.19) and (4.20). Now, according to $\left(\mathrm{P}_{n}\right)$, we have

$$
\left\|\delta \boldsymbol{H}^{k}\right\|_{\boldsymbol{L}_{\mu}^{2}(\Omega)}=\left\|\mu^{\frac{1}{2}} \delta \boldsymbol{H}^{k}\right\|_{\boldsymbol{L}^{2}(\Omega)}=\left\|\mu^{-\frac{1}{2}} \operatorname{curl} \boldsymbol{E}^{k}\right\|_{\boldsymbol{L}^{2}(\Omega)}=\left\|\operatorname{curl} \boldsymbol{E}^{k}\right\|_{\boldsymbol{L}_{\frac{1}{\mu}}^{2}(\Omega)}
$$

and

$$
\left\|\operatorname{curl} \boldsymbol{H}^{k}\right\|_{\boldsymbol{L}^{2}(\Omega)}=\left\|\epsilon \delta \boldsymbol{E}^{k}-\boldsymbol{u}\left(t_{k}\right)+\boldsymbol{J}^{k}\right\|_{\boldsymbol{L}^{2}(\Omega)} \leq \bar{\epsilon}^{\frac{1}{2}}\left\|\delta \boldsymbol{E}^{k}\right\|_{\boldsymbol{L}_{\epsilon}^{2}(\Omega)}+\|\boldsymbol{u}\|_{\mathcal{C}\left([0, T], \boldsymbol{L}^{2}(\Omega)\right)}+|\Omega|^{\frac{1}{2}} C\left(\|\theta\|_{\mathcal{C}\left([0, T], L^{\infty}(\Omega)\right)}\right),
$$

where we have also used (4.13) and (A3). Applying (4.20) to (4.21), (4.22) implies finally (4.15b) and (4.15c).

\section{WeLL-POSEDNESS FOR (QVI)}

For each $n \in \mathbb{N}$, let $\left\{\left(\boldsymbol{E}^{k}, \boldsymbol{H}^{k}, \boldsymbol{J}^{k}\right)\right\}_{k=0}^{n} \subset \boldsymbol{H}_{0}(\mathbf{c u r l}) \times \boldsymbol{X}(\Omega) \times \boldsymbol{L}^{\infty}(\Omega)$ denote a solution to $\left(\mathrm{P}_{n}\right)$ according to Corollary 4.3. Then, we define piecewise linear and continuous in time functions $\widehat{\boldsymbol{E}}_{n}$ and $\widehat{\boldsymbol{H}}_{n}$ as follows:

$$
\begin{aligned}
\widehat{\boldsymbol{E}}_{n}(t) & :=\frac{t-t_{k-1}}{n^{-1} T} \boldsymbol{E}^{k}+\frac{t_{k}-t}{n^{-1} T} \boldsymbol{E}^{k-1} \quad \text { for all } t \in\left[t_{k-1}, t_{k}\right] \text { and all } k=1, \ldots n \\
\widehat{\boldsymbol{H}}_{n}(t) & :=\frac{t-t_{k-1}}{n^{-1} T} \boldsymbol{H}^{k}+\frac{t_{k}-t}{n^{-1} T} \boldsymbol{H}^{k-1} \quad \text { for all } t \in\left[t_{k-1}, t_{k}\right] \text { and all } k=1, \ldots n
\end{aligned}
$$


Here, we recall that $t_{k}=\frac{T}{n} k$. By our construction (5.1), it holds for all $n \in \mathbb{N}$ that $\widehat{\boldsymbol{E}}_{n} \in W^{1, \infty}\left((0, T), \boldsymbol{H}_{0}(\mathbf{c u r l})\right)$ and $\widehat{\boldsymbol{H}}_{n} \in W^{1, \infty}((0, T), \boldsymbol{X}(\Omega))$ with

$$
\begin{array}{lll}
\partial_{t} \widehat{\boldsymbol{E}}_{n}(t)=\frac{\boldsymbol{E}^{k}-\boldsymbol{E}^{k-1}}{n^{-1} T}=\delta \boldsymbol{E}^{k} & \forall t \in\left(t_{k-1}, t_{k}\right) & \forall k=1, \ldots n \\
\partial_{t} \widehat{\boldsymbol{H}}_{n}(t)=\frac{\boldsymbol{H}^{k}-\boldsymbol{H}^{k-1}}{n^{-1} T}=\delta \boldsymbol{H}^{k} & \forall t \in\left(t_{k-1}, t_{k}\right) & \forall k=1, \ldots n .
\end{array}
$$

Next, we introduce piecewise constant in time functions $\boldsymbol{E}_{n}, \boldsymbol{H}_{n}, \boldsymbol{J}_{n}, \boldsymbol{u}_{n}$ and $\theta_{n}$ :

$$
\begin{cases}\boldsymbol{E}_{n}(t):=\boldsymbol{E}^{k} & \text { for all } t \in\left(t_{k-1}, t_{k}\right] \text { and all } k=1, \ldots n \\ \boldsymbol{H}_{n}(t):=\boldsymbol{H}^{k} & \text { for all } t \in\left(t_{k-1}, t_{k}\right] \text { and all } k=1, \ldots n \\ \boldsymbol{J}_{n}(t):=\boldsymbol{J}^{k} & \text { for all } t \in\left(t_{k-1}, t_{k}\right] \text { and all } k=1, \ldots n \\ \boldsymbol{u}_{n}(t):=\boldsymbol{u}\left(t_{k}\right) & \text { for all } t \in\left(t_{k-1}, t_{k}\right] \text { and all } k=1, \ldots n \\ \theta_{n}(t):=\theta\left(t_{k}\right) & \text { for all } t \in\left(t_{k-1}, t_{k}\right] \text { and all } k=1, \ldots n\end{cases}
$$

We note that Assumption 4.1 guarantees that

$$
\lim _{n \rightarrow \infty}\left\|\boldsymbol{u}_{n}-\boldsymbol{u}\right\|_{L^{2}\left((0, T), L^{2}(\Omega)\right)}=\lim _{n \rightarrow \infty}\left\|\theta_{n}-\theta\right\|_{L^{2}\left((0, T), L^{2}(\Omega)\right)}=0 .
$$

For simplicity, we use the notation

$$
j_{c_{n}}(x, t):=j_{c}\left(x, \theta_{n}(x, t), \boldsymbol{H}_{n}(x, t)\right) \text { for a.e. }(x, t) \in \Omega \times(0, T) \text { and all } n \in \mathbb{N} .
$$

In view of $\left(\mathrm{P}_{n}\right),(5.2),(5.3)$, and (5.5) imply for all $n \in \mathbb{N}$ that

$$
\begin{cases}\epsilon \partial_{t} \widehat{\boldsymbol{E}}_{n}-\operatorname{curl} \boldsymbol{H}_{n}+\boldsymbol{J}_{n}=\boldsymbol{u}_{n} & \\ \mu \partial_{t} \widehat{\boldsymbol{H}}_{n}+\operatorname{curl} \boldsymbol{E}_{n}=0 & \text { for a.e. }(x, t) \in \Omega \times(0, T) \\ \left|\boldsymbol{J}_{n}(x, t)\right| \leq j_{c_{n}}(x, t) & \text { for a.e. }(x, t) \in \Omega \times(0, T) . \\ \boldsymbol{J}_{n}(x, t) \cdot \boldsymbol{E}_{n}(x, t)=j_{c_{n}}(x, t)\left|\boldsymbol{E}_{n}(x, t)\right|\end{cases}
$$

Theorem 5.1. Under Assumptions 2.1 and 4.1, (1.1) admits a unique solution $(\boldsymbol{E}, \boldsymbol{H}, \boldsymbol{J}) \quad \in$ $W^{1, \infty}\left((0, T), \boldsymbol{L}^{2}(\Omega)\right) \cap L^{\infty}\left((0, T), \boldsymbol{H}_{0}(\mathbf{c u r l})\right) \times W^{1, \infty}\left((0, T), \boldsymbol{L}^{2}(\Omega)\right) \cap L^{\infty}((0, T), \boldsymbol{X}(\Omega)) \times L^{\infty}\left((0, T), \boldsymbol{L}^{\infty}(\Omega)\right)$.

Proof. Step 1. By definition, the assumption (A3) and Lemmas 4.5 and 4.6, the sequences

$$
\begin{aligned}
&\left\{\widehat{\boldsymbol{E}}_{n}\right\}_{n=1}^{\infty} \subset W^{1, \infty}\left((0, T), \boldsymbol{L}^{2}(\Omega)\right) \cap \mathcal{C}\left([0, T], \boldsymbol{H}_{0}(\mathbf{c u r l})\right) \\
&\left\{\widehat{\boldsymbol{H}}_{n}\right\}_{n=1}^{\infty} \subset W^{1, \infty}\left((0, T), \boldsymbol{L}^{2}(\Omega)\right) \cap \mathcal{C}([0, T], \boldsymbol{X}(\Omega))\left\{\boldsymbol{E}_{n}\right\}_{n=1}^{\infty} \subset L^{\infty}\left((0, T), \boldsymbol{H}_{0}(\mathbf{c u r l})\right) \\
&\left\{\boldsymbol{H}_{n}\right\}_{n=1}^{\infty} \subset L^{\infty}((0, T), \boldsymbol{X}(\Omega)) \\
& \quad\left\{\boldsymbol{J}_{n}\right\}_{n=1}^{\infty} \subset L^{\infty}\left((0, T), \boldsymbol{L}^{\infty}(\Omega)\right)
\end{aligned}
$$

are bounded. Therefore, after selecting subsequences,

$$
\begin{cases}\widehat{\boldsymbol{E}}_{n} \rightarrow \widehat{\boldsymbol{E}} & \text { weakly star in } L^{\infty}\left((0, T), \boldsymbol{H}_{0}(\mathbf{c u r l})\right) \text { as } n \rightarrow \infty \\ \widehat{\boldsymbol{H}}_{n} \rightarrow \widehat{\boldsymbol{H}} & \text { weakly star in } L^{\infty}((0, T), \boldsymbol{X}(\Omega)) \text { as } n \rightarrow \infty \\ \partial_{t} \widehat{\boldsymbol{E}}_{n} \rightarrow \partial_{t} \widehat{\boldsymbol{E}} & \text { weakly star in } L^{\infty}\left((0, T), \boldsymbol{L}^{2}(\Omega)\right) \text { as } n \rightarrow \infty \\ \partial_{t} \widehat{\boldsymbol{H}}_{n} \rightarrow \partial_{t} \widehat{\boldsymbol{H}} & \text { weakly star in } L^{\infty}\left((0, T), \boldsymbol{L}^{2}(\Omega)\right) \text { as } n \rightarrow \infty \\ \boldsymbol{E}_{n} \rightarrow \boldsymbol{E} & \text { weakly star in } L^{\infty}\left((0, T), \boldsymbol{H}_{0}(\mathbf{c u r l})\right) \text { as } n \rightarrow \infty \\ \boldsymbol{H}_{n} \rightarrow \boldsymbol{H} & \text { weakly star in } L^{\infty}((0, T), \boldsymbol{X}(\Omega)) \text { as } n \rightarrow \infty \\ \boldsymbol{J}_{n} \rightarrow \boldsymbol{J} & \text { weakly star in } L^{\infty}\left((0, T), \boldsymbol{L}^{2}(\Omega)\right) \text { as } n \rightarrow \infty\end{cases}
$$


for some $\widehat{\boldsymbol{E}} \in W^{1, \infty}\left((0, T), \boldsymbol{L}^{2}(\Omega)\right) \cap L^{\infty}\left((0, T), \boldsymbol{H}_{0}(\mathbf{c u r l})\right), \widehat{\boldsymbol{H}} \in W^{1, \infty}\left((0, T), \boldsymbol{L}^{2}(\Omega)\right) \cap L^{\infty}((0, T), \boldsymbol{X}(\Omega))$, $\boldsymbol{E} \in L^{\infty}\left((0, T), \boldsymbol{H}_{0}(\mathbf{c u r l})\right), \boldsymbol{H} \in L^{\infty}((0, T), \boldsymbol{X}(\Omega))$, and $\boldsymbol{J} \in L^{\infty}\left((0, T), \boldsymbol{L}^{\infty}(\Omega)\right)$. Note that in (5.7), we use $L^{1}\left((0, T), \boldsymbol{H}_{0}(\mathbf{c u r l})\right)^{*} \cong L^{\infty}\left((0, T), \boldsymbol{H}_{0}(\mathbf{c u r l})\right)$ and similar identifications for other $L^{1}$-Bochner spaces. According to (5.1)-(5.3) and Lemma 4.6, it holds for all $n \geq \beta$ that

$$
\begin{aligned}
\left\|\widehat{\boldsymbol{E}}_{n}-\boldsymbol{E}_{n}\right\|_{L^{\infty}\left((0, T), \boldsymbol{L}_{\epsilon}^{2}(\Omega)\right)} & \leq \frac{T}{n} \max _{k=1, \ldots, n}\left\|\delta \boldsymbol{E}^{k}\right\|_{\boldsymbol{L}_{\epsilon}^{2}(\Omega)} \leq \frac{T}{n} \sqrt{2 \alpha(1+\beta \exp (\beta))} \\
\left\|\widehat{\boldsymbol{H}}_{n}-\boldsymbol{H}_{n}\right\|_{L^{\infty}\left((0, T), \boldsymbol{L}_{\mu}^{2}(\Omega)\right)} & \leq \frac{T}{n} \max _{k=1, \ldots, n}\left\|\delta \boldsymbol{H}^{k}\right\|_{L_{\mu}^{2}(\Omega)} \leq \frac{T}{n} \sqrt{2 \alpha \exp (\beta)},
\end{aligned}
$$

which yields

$$
\lim _{n \rightarrow \infty}\left\|\widehat{\boldsymbol{E}}_{n}-\boldsymbol{E}_{n}\right\|_{L^{\infty}\left((0, T), \boldsymbol{L}^{2}(\Omega)\right)}=\lim _{n \rightarrow \infty}\left\|\widehat{\boldsymbol{H}}_{n}-\boldsymbol{H}_{n}\right\|_{L^{\infty}\left((0, T), \boldsymbol{L}^{2}(\Omega)\right)}=0 .
$$

Thus, the properties (5.7) and (5.8) imply

$$
\left\{\begin{array}{l}
\boldsymbol{E}=\widehat{\boldsymbol{E}} \in W^{1, \infty}\left((0, T), \boldsymbol{L}^{2}(\Omega)\right) \cap L^{\infty}\left((0, T), \boldsymbol{H}_{0}(\text { curl })\right) \\
\boldsymbol{H}=\widehat{\boldsymbol{H}} \in W^{1, \infty}\left((0, T), \boldsymbol{L}^{2}(\Omega)\right) \cap L^{\infty}((0, T), \boldsymbol{X}(\Omega)) .
\end{array}\right.
$$

Altogether, in view of (5.4), (5.6), (5.7), and (5.9), the weak limit $(\boldsymbol{E}, \boldsymbol{H}, \boldsymbol{J})$ satisfies

$$
\left\{\begin{array}{l}
\epsilon \partial_{t} \boldsymbol{E}-\operatorname{curl} \boldsymbol{H}+\boldsymbol{J}=\boldsymbol{u} \\
\mu \partial_{t} \boldsymbol{H}+\operatorname{curl} \boldsymbol{E}=0
\end{array}\right.
$$

Step 2. Thanks to (5.9), possibly after a modification on a set of $[0, T]$ with measure zero, it holds that $\boldsymbol{E}, \boldsymbol{H} \in \mathcal{C}\left([0, T], \boldsymbol{L}^{2}(\Omega)\right)$. On the other hand, as $\boldsymbol{X}(\Omega) \hookrightarrow \boldsymbol{L}^{2}(\Omega)$ is compact, the Aubin-Lions lemma yields the compactness of the embedding $H^{1}\left((0, T), \boldsymbol{L}^{2}(\Omega)\right) \cap L^{\infty}((0, T), \boldsymbol{X}(\Omega)) \hookrightarrow \mathcal{C}\left([0, T], \boldsymbol{L}^{2}(\Omega)\right)$. For this reason,

$$
\widehat{\boldsymbol{H}}_{n} \rightarrow \boldsymbol{H} \quad \text { in } \mathcal{C}\left([0, T], \boldsymbol{L}^{2}(\Omega)\right) \text { as } n \rightarrow \infty .
$$

In particular, as $\widehat{\boldsymbol{H}}_{n}(0)=\boldsymbol{H}_{0}$ for all $n \in \mathbb{N}$, the above convergence implies

$$
\boldsymbol{H}(0)=\boldsymbol{H}_{0}
$$

We underline that $\boldsymbol{H}_{0}(\mathbf{c u r l}) \hookrightarrow \boldsymbol{L}^{2}(\Omega)$ is not compact so that the convergence of $\left\{\widehat{\boldsymbol{E}}_{n}\right\}_{n=1}^{\infty}$ in $\mathcal{C}\left([0, T], \boldsymbol{L}^{2}(\Omega)\right)$ cannot be expected. However, we will show that

$$
\widehat{\boldsymbol{E}}_{n}(T) \rightarrow \boldsymbol{E}(T) \quad \text { weakly in } \boldsymbol{L}^{2}(\Omega) \text { as } n \rightarrow \infty
$$

holds after selecting a subsequence, and

$$
\boldsymbol{E}(0)=\boldsymbol{E}_{0}
$$

By virtue of Lemma 4.5, the sequence $\left\{\boldsymbol{E}^{n}\right\}_{n=1}^{\infty} \subset \boldsymbol{L}^{2}(\Omega)$ is bounded. Thus, after selecting a subsequence, it holds that

$$
\boldsymbol{E}^{n} \rightarrow \boldsymbol{E}_{T} \quad \text { weakly in } \boldsymbol{L}^{2}(\Omega) \text { as } n \rightarrow \infty
$$

for some $\boldsymbol{E}_{T} \in \boldsymbol{L}^{2}(\Omega)$. We introduce the function $\varphi:[0, T] \rightarrow[0,1], t \mapsto \frac{t}{T}$. By definition and employing the classical integration by parts formula, we have

$$
\begin{aligned}
\int_{\Omega} \epsilon \boldsymbol{E}^{n} \cdot \boldsymbol{v} \mathrm{d} x & =\int_{\Omega} \epsilon \widehat{\boldsymbol{E}}_{n}(T) \cdot \boldsymbol{v} \mathrm{d} x \varphi(T)-\int_{\Omega} \epsilon \widehat{\boldsymbol{E}}_{n}(0) \cdot \boldsymbol{v} \mathrm{d} x \varphi(0) \\
& =\int_{0}^{T}\left(\int_{\Omega} \epsilon \partial_{t} \widehat{\boldsymbol{E}}_{n}(t) \cdot \boldsymbol{v} \mathrm{d} x \varphi(t)+\int_{\Omega} \epsilon \widehat{\boldsymbol{E}}_{n}(t) \cdot \boldsymbol{v} \mathrm{d} x \varphi^{\prime}(t)\right) \mathrm{d} t \quad \forall n \in \mathbb{N}, \quad \forall \boldsymbol{v} \in \boldsymbol{L}^{2}(\Omega) .
\end{aligned}
$$


Then, passing to the limit $n \rightarrow \infty$, we obtain from (5.7), (5.9), and (5.15) that

$$
\begin{aligned}
\int_{\Omega} \epsilon \boldsymbol{E}_{T} \cdot \boldsymbol{v} \mathrm{d} x & =\int_{0}^{T}\left(\int_{\Omega} \epsilon \partial_{t} \boldsymbol{E}(t) \cdot \boldsymbol{v} \mathrm{d} x \varphi(t)+\int_{\Omega} \epsilon \boldsymbol{E}(t) \cdot \boldsymbol{v} \mathrm{d} x \varphi^{\prime}(t)\right) \mathrm{d} t \\
& =\int_{\Omega} \epsilon \boldsymbol{E}(T) \cdot \boldsymbol{v} \mathrm{d} x \varphi(T)-\int_{\Omega} \epsilon \boldsymbol{E}(0) \cdot \boldsymbol{v} \mathrm{d} x \varphi(0)=\int_{\Omega} \epsilon \boldsymbol{E}(T) \cdot \boldsymbol{v} \mathrm{d} x \quad \forall \boldsymbol{v} \in \boldsymbol{L}^{2}(\Omega),
\end{aligned}
$$

from which it follows that $\boldsymbol{E}_{T}=\boldsymbol{E}(T)$, and consequently

$$
\widehat{\boldsymbol{E}}_{n}(T)=\boldsymbol{E}^{n} \rightarrow \boldsymbol{E}_{T}=\boldsymbol{E}(T) \quad \text { weakly in } \boldsymbol{L}^{2}(\Omega) \text { as } n \rightarrow \infty .
$$

Similarly, introducing the function $\psi:[0, T] \rightarrow[-1,0], t \mapsto \frac{t-T}{T}$, we deduce that

$$
\begin{aligned}
\int_{\Omega} \epsilon \boldsymbol{E}_{0} \cdot \boldsymbol{v} \mathrm{d} x & =\int_{\Omega} \epsilon \widehat{\boldsymbol{E}}_{n}(T) \cdot \boldsymbol{v} \mathrm{d} x \psi(T)-\int_{\Omega} \epsilon \widehat{\boldsymbol{E}}_{n}(0) \cdot \boldsymbol{v} \mathrm{d} x \psi(0) \\
& =\int_{0}^{T}\left(\int_{\Omega} \epsilon \partial_{t} \widehat{\boldsymbol{E}}_{n}(t) \cdot \boldsymbol{v} \mathrm{d} x \psi(t)+\int_{\Omega} \epsilon \widehat{\boldsymbol{E}}_{n}(t) \cdot \boldsymbol{v} \mathrm{d} x \psi^{\prime}(t)\right) \mathrm{d} t \\
& \rightarrow \int_{0}^{T}\left(\int_{\Omega} \epsilon \partial_{t} \boldsymbol{E}(t) \cdot \boldsymbol{v} \mathrm{d} x \psi(t)+\int_{\Omega} \epsilon \boldsymbol{E}(t) \cdot \boldsymbol{v} \mathrm{d} x \psi^{\prime}(t)\right) \mathrm{d} t \\
& =\int_{\Omega} \epsilon \boldsymbol{E}(T) \cdot \boldsymbol{v} \mathrm{d} x \psi(T)-\int_{\Omega} \epsilon \boldsymbol{E}(0) \cdot \boldsymbol{v} \mathrm{d} x \psi(0)=\int_{\Omega} \epsilon \boldsymbol{E}(0) \cdot \boldsymbol{v} \mathrm{d} x \quad \forall \boldsymbol{v} \in \boldsymbol{L}^{2}(\Omega),
\end{aligned}
$$

from which it follows that $\boldsymbol{E}(0)=\boldsymbol{E}_{0}$. In conclusion, the conditions (5.13) and (5.14) are valid.

Step 3. We verify the conditions

$$
\begin{array}{ll}
|\boldsymbol{J}(x, t)| \leq j_{c}(x, \theta(x, t), \boldsymbol{H}(x, t)) & \text { for a.e. }(x, t) \in \Omega \times(0, T) \\
\boldsymbol{J}(x, t) \cdot \boldsymbol{E}(x, t)=j_{c}(x, \theta(x, t), \boldsymbol{H}(x, t))|\boldsymbol{E}(x, t)| & \text { for a.e. }(x, t) \in \Omega \times(0, T) .
\end{array}
$$

The convergence properties (5.8) and (5.11) imply

$$
\boldsymbol{H}_{n} \rightarrow \boldsymbol{H} \text { in } L^{\infty}\left((0, T), \boldsymbol{L}^{2}(\Omega)\right) \text { as } n \rightarrow \infty .
$$

Then, in view of (A4), (5.4), (5.5), and (5.17), we obtain

$$
\begin{aligned}
& \lim _{n \rightarrow \infty} \int_{0}^{T} \int_{\Omega}\left|j_{c n}(x, t)-j_{c}(x, \theta(x, t), \boldsymbol{H}(x, t))\right|^{2} \mathrm{~d} x \mathrm{~d} t \\
& \quad \leq \lim _{n \rightarrow \infty} 2 L\left(\|\theta\|_{\mathcal{C}\left([0, T], L^{\infty}(\Omega)\right)}\right)^{2}\left(\left\|\theta_{n}-\theta\right\|_{L^{2}\left((0, T), L^{2}(\Omega)\right)}^{2}+\left\|\boldsymbol{H}_{n}-\boldsymbol{H}\right\|_{L^{2}\left((0, T), L^{2}(\Omega)\right)}^{2}\right)=0 .
\end{aligned}
$$

In other words,

$$
j_{c_{n}} \rightarrow j_{c}(\cdot, \theta, \boldsymbol{H}) \quad \text { in } L^{2}\left((0, T), \boldsymbol{L}^{2}(\Omega)\right) \text { as } n \rightarrow \infty .
$$

Now, suppose that there exists a Lebesgue measurable set $Q \subset \Omega \times(0, T)$ of non-zero measure such that

$$
j_{c}(x, \theta(x, t), \boldsymbol{H}(x, t))<|\boldsymbol{J}(x, t)| \text { for a.e. }(x, t) \in Q .
$$

This inequality together with the weak convergence (5.7) and the convergence (5.18) leads to a contradiction:

$$
\begin{aligned}
\iint_{Q} j_{c}(x, \theta(x, t), \boldsymbol{H}(x, t)) \mathrm{d} x \mathrm{~d} t<\iint_{Q}|\boldsymbol{J}(x, t)| \mathrm{d} x \mathrm{~d} t \\
\underbrace{=}_{(5.7)} \lim _{n \rightarrow \infty} \iint_{Q} \boldsymbol{J}_{n}(x, t) \cdot \frac{\boldsymbol{J}(x, t)}{|\boldsymbol{J}(x, t)|} \mathrm{d} x \mathrm{~d} t \underbrace{\leq}_{(5.6)} \lim _{n \rightarrow \infty} \iint_{Q} j_{c_{n}}(x, t) \mathrm{d} x \mathrm{~d} t \underbrace{=}_{(5.18)} \iint_{Q} j_{c}(x, \theta(x, t), \boldsymbol{H}(x, t)) \mathrm{d} x \mathrm{~d} t .
\end{aligned}
$$


We come therefore to the conclusion that

$$
|\boldsymbol{J}(x, t)| \leq j_{c}(x, \theta(x, t), \boldsymbol{H}(x, t)) \quad \text { for a.e. }(x, t) \in \Omega \times(0, T) .
$$

Next, let $k \in \mathbb{N}$. The convergence (5.18) together with the weak convergence (5.7) implies

$$
\begin{aligned}
\int_{0}^{T} \int_{\Omega} j_{c}(x, \theta(x, t), \boldsymbol{H}(x, t)) \frac{|\boldsymbol{E}(x, t)|^{2}}{|\boldsymbol{E}(x, t)|+k^{-1}} \mathrm{~d} x \mathrm{~d} t & =\lim _{n \rightarrow \infty} \int_{0}^{T} \int_{\Omega} j_{c_{n}}(x, t) \frac{\boldsymbol{E}(x, t)}{|\boldsymbol{E}(x, t)|+k^{-1}} \cdot \boldsymbol{E}_{n}(x, t) \mathrm{d} x \mathrm{~d} t \\
& =\liminf _{n \rightarrow \infty} \int_{0}^{T} \int_{\Omega} j_{c_{n}}(x, t) \frac{\boldsymbol{E}(x, t)}{|\boldsymbol{E}(x, t)|+k^{-1}} \cdot \boldsymbol{E}_{n}(x, t) \mathrm{d} x \mathrm{~d} t \\
& \leq \liminf _{n \rightarrow \infty} \int_{0}^{T} \int_{\Omega} j_{c_{n}}(x, t)\left|\boldsymbol{E}_{n}(x, t)\right| \mathrm{d} x \mathrm{~d} t \\
& \underbrace{=}_{(5.6)} \liminf _{n \rightarrow \infty} \int_{0}^{T} \int_{\Omega} \boldsymbol{J}_{n}(x, t) \cdot \boldsymbol{E}_{n}(x, t) \mathrm{d} x \mathrm{~d} t .
\end{aligned}
$$

Passing to the limit $k \rightarrow \infty$, the monotone convergence theorem implies

$$
\int_{0}^{T} \int_{\Omega} j_{c}(x, \theta(x, t), \boldsymbol{H}(x, t))|\boldsymbol{E}(x, t)| \mathrm{d} x \mathrm{~d} t \leq \liminf _{n \rightarrow \infty} \int_{0}^{T} \int_{\Omega} \boldsymbol{J}_{n}(x, t) \cdot \boldsymbol{E}_{n}(x, t) \mathrm{d} x \mathrm{~d} t .
$$

Let us investigate the right-hand side of the above inequality. According to (5.10),

$$
\left(\partial_{t} \boldsymbol{E}(t), \boldsymbol{E}(t)\right)_{\boldsymbol{L}_{\epsilon}^{2}(\Omega)}+\left(\partial_{t} \boldsymbol{H}(t), \boldsymbol{H}(t)\right)_{\boldsymbol{L}_{\mu}^{2}(\Omega)}+(\boldsymbol{J}(t), \boldsymbol{E}(t))_{\boldsymbol{L}^{2}(\Omega)}=(\boldsymbol{u}(t), \boldsymbol{E}(t))_{\boldsymbol{L}^{2}(\Omega)}
$$

holds for a.e. $t \in(0, T)$. Integrating this identity over the time interval $[0, T]$ and employing (5.12) as well as (5.14), we obtain

$$
\|\boldsymbol{E}(T)\|_{\boldsymbol{L}_{\epsilon}^{2}(\Omega)}^{2}+\|\boldsymbol{H}(T)\|_{\boldsymbol{L}_{\mu}^{2}(\Omega)}^{2}+2 \int_{0}^{T}(\boldsymbol{J}(t), \boldsymbol{E}(t))_{\boldsymbol{L}^{2}(\Omega)} \mathrm{d} t=\left\|\boldsymbol{E}_{0}\right\|_{\boldsymbol{L}_{\epsilon}^{2}(\Omega)}^{2}+\left\|\boldsymbol{H}_{0}\right\|_{\boldsymbol{L}_{\mu}^{2}(\Omega)}^{2}+2 \int_{0}^{T}(\boldsymbol{u}(t), \boldsymbol{E}(t))_{\boldsymbol{L}^{2}(\Omega)} \mathrm{d} t .
$$

In a similar manner, we deduce from (5.6) that

$$
\begin{aligned}
\left\|\widehat{\boldsymbol{E}}_{n}(T)\right\|_{\boldsymbol{L}_{\epsilon}^{2}(\Omega)}^{2} & +\left\|\widehat{\boldsymbol{H}}_{n}(T)\right\|_{\boldsymbol{L}_{\mu}^{2}(\Omega)}^{2}+2 \int_{0}^{T}\left(\boldsymbol{J}_{n}(t), \boldsymbol{E}_{n}(t)\right)_{\boldsymbol{L}^{2}(\Omega)} \mathrm{d} t=\left\|\boldsymbol{E}_{0}\right\|_{\boldsymbol{L}_{\epsilon}^{2}(\Omega)}^{2}+\left\|\boldsymbol{H}_{0}\right\|_{\boldsymbol{L}_{\mu}^{2}(\Omega)}^{2} \\
& +2 \int_{0}^{T}\left(\boldsymbol{H}_{n}(t), \operatorname{curl} \widehat{\boldsymbol{E}}_{n}(t)\right)_{\boldsymbol{L}^{2}(\Omega)}-\left(\widehat{\boldsymbol{H}}_{n}(t), \operatorname{curl} \boldsymbol{E}_{n}(t)\right)_{\boldsymbol{L}^{2}(\Omega)} \\
& +\left(\boldsymbol{u}_{n}(t), \widehat{\boldsymbol{E}}_{n}(t)\right)_{\boldsymbol{L}^{2}(\Omega)}+\left(\boldsymbol{J}_{n}(t), \boldsymbol{E}_{n}(t)-\widehat{\boldsymbol{E}}_{n}(t)\right)_{\boldsymbol{L}^{2}(\Omega)} \mathrm{d} t .
\end{aligned}
$$

Applying (5.21) and (5.22) leads to

$$
2 \int_{0}^{T}\left(\boldsymbol{J}_{n}(t), \boldsymbol{E}_{n}(t)\right)_{\boldsymbol{L}^{2}(\Omega)} \mathrm{d} t=\mathrm{I}_{n}+\mathrm{II}_{n}+2 \int_{0}^{T}(\boldsymbol{J}(t)-\boldsymbol{u}(t), \boldsymbol{E}(t))_{\boldsymbol{L}^{2}(\Omega)} \mathrm{d} t
$$

with

$$
\begin{aligned}
\mathrm{I}_{n}:= & \|\boldsymbol{E}(T)\|_{\boldsymbol{L}_{\epsilon}^{2}(\Omega)}^{2}+\|\boldsymbol{H}(T)\|_{\boldsymbol{L}_{\mu}^{2}(\Omega)}^{2}-\left\|\widehat{\boldsymbol{E}}_{n}(T)\right\|_{\boldsymbol{L}_{\epsilon}^{2}(\Omega)}^{2}-\left\|\widehat{\boldsymbol{H}}_{n}(T)\right\|_{\boldsymbol{L}_{\mu}^{2}(\Omega)}^{2} \\
\mathrm{II}_{n}:= & 2 \int_{0}^{T}\left(\boldsymbol{H}_{n}(t), \operatorname{curl} \widehat{\boldsymbol{E}}_{n}(t)\right)_{\boldsymbol{L}^{2}(\Omega)}-\left(\widehat{\boldsymbol{H}}_{n}(t), \operatorname{curl} \boldsymbol{E}_{n}(t)\right)_{\boldsymbol{L}^{2}(\Omega)} \\
& +\left(\boldsymbol{u}_{n}(t), \widehat{\boldsymbol{E}}_{n}(t)\right)_{\boldsymbol{L}^{2}(\Omega)}+\left(\boldsymbol{J}_{n}(t), \boldsymbol{E}_{n}(t)-\widehat{\boldsymbol{E}}_{n}(t)\right)_{\boldsymbol{L}^{2}(\Omega)} \mathrm{d} t .
\end{aligned}
$$


As a consequence of (5.11) and (5.13), the first term $\mathrm{I}_{n}$ satisfies

$$
\begin{aligned}
\limsup _{n \rightarrow \infty} I_{n} & \leq\|\boldsymbol{E}(T)\|_{\boldsymbol{L}_{\epsilon}^{2}(\Omega)}^{2}+\|\boldsymbol{H}(T)\|_{\boldsymbol{L}_{\mu}^{2}(\Omega)}^{2}+\limsup _{n \rightarrow \infty}\left(-\left\|\widehat{\boldsymbol{E}}_{n}(T)\right\|_{\boldsymbol{L}_{\epsilon}^{2}(\Omega)}^{2}-\left\|\widehat{\boldsymbol{H}}_{n}(T)\right\|_{\boldsymbol{L}_{\mu}^{2}(\Omega)}^{2}\right) \\
& \leq\|\boldsymbol{E}(T)\|_{\boldsymbol{L}_{\epsilon}^{2}(\Omega)}^{2}-\liminf _{n \rightarrow \infty}\left\|\widehat{\boldsymbol{E}}_{n}(T)\right\|_{\boldsymbol{L}_{\epsilon}^{2}(\Omega)}^{2} \leq 0 .
\end{aligned}
$$

Furthermore, thanks to (5.4), (5.7), (5.8), (5.9), (5.11), and (5.17), passing to the limit $n \rightarrow \infty$ in the second term $\mathrm{II}_{n}$ leads to

$$
\lim _{n \rightarrow \infty} \mathrm{II}_{n}=2 \int_{0}^{T}(\boldsymbol{u}(t), \boldsymbol{E}(t))_{\boldsymbol{L}^{2}(\Omega)} \mathrm{d} t .
$$

Invoking the above convergence results, we obtain from (5.23) that

$$
\liminf _{n \rightarrow \infty} \int_{0}^{T}\left(\boldsymbol{J}_{n}(t), \boldsymbol{E}_{n}(t)\right)_{\boldsymbol{L}^{2}(\Omega)} \mathrm{d} t \leq \limsup _{n \rightarrow \infty} \int_{0}^{T}\left(\boldsymbol{J}_{n}(t), \boldsymbol{E}_{n}(t)\right)_{\boldsymbol{L}^{2}(\Omega)} \mathrm{d} t \leq \int_{0}^{T}(\boldsymbol{J}(t), \boldsymbol{E}(t))_{\boldsymbol{L}^{2}(\Omega)} \mathrm{d} t .
$$

As a result of (5.24) and (5.20),

$$
\int_{0}^{T} \int_{\Omega} j_{c}(x, \theta(x, t), \boldsymbol{H}(x, t))|\boldsymbol{E}(x, t)|-\boldsymbol{J}(x, t) \cdot \boldsymbol{E}(x, t) \mathrm{d} x \mathrm{~d} t \leq 0 .
$$

On the other hand, thanks to (5.19),

$$
j_{c}(x, \theta(x, t), \boldsymbol{H}(x, t))|\boldsymbol{E}(x, t)|-\boldsymbol{J}(x, t) \cdot \boldsymbol{E}(x, t) \geq 0 \quad \text { for a.e. }(x, t) \in \Omega \times(0, T) .
$$

Combining the above two inequalities, we arrive at

$$
\boldsymbol{J}(x, t) \cdot \boldsymbol{E}(x, t)=j_{c}(x, \theta(x, t), \boldsymbol{H}(x, t))|\boldsymbol{E}(x, t)| \text { for a.e. }(x, t) \in \Omega \times(0, T) .
$$

In conclusion, (5.16) is valid.

Collecting all the previously proven results (5.10), (5.12), (5.14) and (5.16), we come to the conclusion that the weak limit $(\boldsymbol{E}, \boldsymbol{H}, \boldsymbol{J}) \in W^{1, \infty}\left((0, T), \boldsymbol{L}^{2}(\Omega)\right) \cap L^{\infty}\left((0, T), \boldsymbol{H}_{0}(\mathbf{c u r l})\right) \times W^{1, \infty}\left((0, T), \boldsymbol{L}^{2}(\Omega)\right) \cap$ $L^{\infty}((0, T), \boldsymbol{X}(\Omega)) \times L^{\infty}\left((0, T), \boldsymbol{L}^{\infty}(\Omega)\right)$ is a solution to $(1.1)$.

Step 4. We complete the proof by verifying the uniqueness. Suppose that $(\widetilde{\boldsymbol{E}}, \widetilde{\boldsymbol{H}}, \widetilde{\boldsymbol{J}}) \in W^{1, \infty}\left((0, T), \boldsymbol{L}^{2}(\Omega)\right) \cap$ $L^{\infty}\left((0, T), \boldsymbol{H}_{0}(\mathbf{c u r l})\right) \times W^{1, \infty}\left((0, T), \boldsymbol{L}^{2}(\Omega)\right) \cap L^{\infty}((0, T), \boldsymbol{X}(\Omega)) \times L^{\infty}\left((0, T), \boldsymbol{L}^{\infty}(\Omega)\right)$ is another solution of (1.1), i.e., it satisfies

$$
\begin{array}{ll}
\epsilon \partial_{t} \widetilde{\boldsymbol{E}}-\operatorname{curl} \widetilde{\boldsymbol{H}}+\widetilde{\boldsymbol{J}}=\boldsymbol{u} & \text { in } \Omega \times(0, T) \\
\mu \partial_{t} \widetilde{\boldsymbol{H}}+\operatorname{curl} \widetilde{\boldsymbol{E}}=0 & \text { in } \Omega \times(0, T) \\
\widetilde{\boldsymbol{E}}(\cdot, 0)=\boldsymbol{E}_{0}, \widetilde{\boldsymbol{H}}(\cdot, 0)=\boldsymbol{H}_{0} & \text { in } \Omega \\
\widetilde{\boldsymbol{J}}(x, t) \cdot \widetilde{\boldsymbol{E}}(x, t)=j_{c}(x, \theta(x, t), \widetilde{\boldsymbol{H}}(x, t))|\widetilde{\boldsymbol{E}}(x, t)| & \text { for a.e. }(x, t) \in \Omega \times(0, T) \\
|\widetilde{\boldsymbol{J}}(x, t)| \leq j_{c}(x, \theta(x, t), \widetilde{\boldsymbol{H}}(x, t)) & \text { for a.e. }(x, t) \in \Omega \times(0, T) .
\end{array}
$$

In view of (5.25) and (1.1), $(\boldsymbol{e}, \boldsymbol{h}):=(\widetilde{\boldsymbol{E}}-\boldsymbol{E}, \widetilde{\boldsymbol{H}}-\boldsymbol{H})$ fullfils

$$
\left(\partial_{t} \boldsymbol{e}(t), \boldsymbol{e}(t)\right)_{\boldsymbol{L}_{\epsilon}^{2}(\Omega)}+\left(\partial_{t} \boldsymbol{h}(t), \boldsymbol{h}(t)\right)_{\boldsymbol{L}_{\mu}^{2}(\Omega)}+(\widetilde{\boldsymbol{J}}(t)-\boldsymbol{J}(t), \boldsymbol{e}(t))_{\boldsymbol{L}^{2}(\Omega)}=0 \quad \text { a.e. } t \in(0, T) .
$$

Since $\boldsymbol{e}(0)=\boldsymbol{h}(0)=0$, integrating this equality over the time interval yields

$$
\|\boldsymbol{e}(t)\|_{\boldsymbol{L}_{\epsilon}^{2}(\Omega)}^{2}+\|\boldsymbol{h}(t)\|_{\boldsymbol{L}_{\mu}^{2}(\Omega)}^{2}=2 \int_{0}^{t}(\boldsymbol{J}(s)-\widetilde{\boldsymbol{J}}(s), \boldsymbol{e}(s))_{\boldsymbol{L}^{2}(\Omega)} \mathrm{d} s \quad \forall t \in[0, T] .
$$


Invoking the conditions for $\boldsymbol{J}$ and $\widetilde{\boldsymbol{J}}$, the right-hand side of the above equality satisfies

$$
\begin{aligned}
& \int_{0}^{t}(\boldsymbol{J}(s)-\widetilde{\boldsymbol{J}}(s), \boldsymbol{e}(s))_{\boldsymbol{L}^{2}(\Omega)} \mathrm{d} s \\
& =\int_{0}^{t} \int_{\Omega} \boldsymbol{J}(x, s) \cdot \widetilde{\boldsymbol{E}}(x, s)-j_{c}(x, \theta(x, s), \boldsymbol{H}(x, s))|\boldsymbol{E}(x, s)| \\
& \quad+\widetilde{\boldsymbol{J}}(x, s) \cdot \boldsymbol{E}(x, s)-j_{c}(x, \theta(x, s), \widetilde{\boldsymbol{H}}(x, s))|\widetilde{\boldsymbol{E}}(x, s)| \mathrm{d} x \mathrm{~d} s \\
& \leq \int_{0}^{t} \int_{\Omega} j_{c}(x, \theta(x, s), \boldsymbol{H}(x, s))(|\widetilde{\boldsymbol{E}}(x, s)|-|\boldsymbol{E}(x, s)|)+j_{c}(x, \theta(x, s), \widetilde{\boldsymbol{H}}(x, s))(|\boldsymbol{E}(x, s)|-|\widetilde{\boldsymbol{E}}(x, s)|) \mathrm{d} x \mathrm{~d} s \\
& \leq \int_{0}^{t} \int_{\Omega}\left(j_{c}(x, \theta(x, s), \boldsymbol{H}(x, s))-j_{c}(x, \theta(x, s), \widetilde{\boldsymbol{H}}(x, s))\right)|\boldsymbol{e}(x, s)| \mathrm{d} x \mathrm{~d} s \\
& \underbrace{\leq}_{(\mathrm{A} 4)} L\left(\|\theta\|_{\mathcal{C}\left([0, T], L^{\infty}(\Omega)\right)}\right) \int_{0}^{t} \int_{\Omega}|\boldsymbol{h}(x, s)||\boldsymbol{e}(x, s)| \mathrm{d} x \mathrm{~d} s \\
& \leq L\left(\|\theta\|_{\mathcal{C}\left([0, T], L^{\infty}(\Omega)\right)}\right)\left(\frac{1}{2 \epsilon} \int_{0}^{t}\|\boldsymbol{e}(s)\|_{\boldsymbol{L}_{\epsilon}^{2}(\Omega)}^{2} \mathrm{~d} s+\frac{1}{2 \mu} \int_{0}^{t}\|\boldsymbol{h}(s)\|_{\boldsymbol{L}_{\mu}^{2}(\Omega)}^{2} \mathrm{~d} s\right) \quad \forall t \in[0, T] .
\end{aligned}
$$

Applying this inequality to (5.26), the Gronwall lemma yields that $\boldsymbol{e} \equiv \boldsymbol{h} \equiv 0$.

Corollary 5.2. Let Assumptions 2.1 and 4.1 be satisfied. Then, the hyperbolic Maxwell quasi-variational inequality

$$
\left\{\begin{array}{l}
\int_{\Omega} \epsilon \partial_{t} \boldsymbol{E}(t) \cdot(\boldsymbol{v}-\boldsymbol{E}(t))+\mu \partial_{t} \boldsymbol{H}(t) \cdot(\boldsymbol{w}-\boldsymbol{H}(t))+\operatorname{curl} \boldsymbol{E}(t) \cdot \boldsymbol{w}-\operatorname{curl} \boldsymbol{H}(t) \cdot \boldsymbol{v} \mathrm{d} x \\
+\int_{\Omega} j_{c}(x, \theta(x, t), \boldsymbol{H}(x, t))(|\boldsymbol{v}(x)|-|\boldsymbol{E}(x, t)|) \mathrm{d} x \geq \int_{\Omega} \boldsymbol{u}(t) \cdot(\boldsymbol{v}-\boldsymbol{E}(t)) \mathrm{d} x \\
\text { for } \text { a.e. } t \in(0, T) \text { and all }(\boldsymbol{v}, \boldsymbol{w}) \in \boldsymbol{L}^{2}(\Omega) \times \boldsymbol{L}^{2}(\Omega) \\
(\boldsymbol{E}, \boldsymbol{H})(0)=\left(\boldsymbol{E}_{0}, \boldsymbol{H}_{0}\right)
\end{array}\right.
$$

admits a unique solution

$$
(\boldsymbol{E}, \boldsymbol{H}) \in W^{1, \infty}\left((0, T), \boldsymbol{L}^{2}(\Omega)\right) \cap L^{\infty}\left((0, T), \boldsymbol{H}_{0}(\mathbf{c u r l})\right) \times W^{1, \infty}\left((0, T), \boldsymbol{L}^{2}(\Omega)\right) \cap L^{\infty}((0, T), \boldsymbol{X}(\Omega)),
$$

which is exactly the unique solution to (1.1).

Proof. Uniqueness. Suppose that

$\left(\boldsymbol{E}^{1}, \boldsymbol{H}^{1}\right),\left(\boldsymbol{E}^{2}, \boldsymbol{H}^{2}\right) \in W^{1, \infty}\left((0, T), \boldsymbol{L}^{2}(\Omega)\right) \cap L^{\infty}\left((0, T), \boldsymbol{H}_{0}(\mathbf{c u r l})\right) \times W^{1, \infty}\left((0, T), \boldsymbol{L}^{2}(\Omega)\right) \cap L^{\infty}((0, T), \boldsymbol{X}(\Omega))$ are solutions to (QVI), and let $(\boldsymbol{e}, \boldsymbol{h}):=\left(\boldsymbol{E}^{1}-\boldsymbol{E}^{2}, \boldsymbol{H}^{1}-\boldsymbol{H}^{2}\right)$. Setting $(\boldsymbol{v}, \boldsymbol{w})=\left(\boldsymbol{E}^{2}, \boldsymbol{H}^{2}\right)(t)($ resp. $(\boldsymbol{v}, \boldsymbol{w})=$ $\left.\left(\boldsymbol{E}^{1}, \boldsymbol{H}^{1}\right)(t)\right)$ in (QVI) for $\left(\boldsymbol{E}^{1}, \boldsymbol{H}^{1}\right)$ (resp. in (QVI) for $\left.\left(\boldsymbol{E}^{2}, \boldsymbol{H}^{2}\right)\right)$ yields

$$
\begin{aligned}
\int_{\Omega}- & \epsilon \partial_{t} \boldsymbol{E}^{1}(t) \cdot \boldsymbol{e}(t)-\mu \partial_{t} \boldsymbol{H}^{1}(t) \cdot \boldsymbol{h}(t)+\operatorname{curl} \boldsymbol{E}^{1}(t) \cdot \boldsymbol{H}^{2}(t)-\operatorname{curl} \boldsymbol{H}^{1}(t) \cdot \boldsymbol{E}^{2}(t) \mathrm{d} x \\
& +\int_{\Omega} j_{c}\left(x, \theta(x, t), \boldsymbol{H}^{1}(x, t)\right)\left(\left|\boldsymbol{E}^{2}(x, t)\right|-\left|\boldsymbol{E}^{1}(x, t)\right|\right) \mathrm{d} x \geq \int_{\Omega}-\boldsymbol{u}(t) \cdot \boldsymbol{e}(t) \mathrm{d} x \quad \text { for a.e. } t \in(0, T)
\end{aligned}
$$


and

$$
\begin{aligned}
& \int_{\Omega} \epsilon \partial_{t} \boldsymbol{E}^{2}(t) \cdot \boldsymbol{e}(t)+\mu \partial_{t} \boldsymbol{H}^{2}(t) \cdot \boldsymbol{h}(t)+\operatorname{curl} \boldsymbol{E}^{2}(t) \cdot \boldsymbol{H}^{1}(t)-\operatorname{curl} \boldsymbol{H}^{2}(t) \cdot \boldsymbol{E}^{1}(t) \mathrm{d} x \\
& \quad+\int_{\Omega} j_{c}\left(x, \theta(x, t), \boldsymbol{H}^{2}(x, t)\right)\left(\left|\boldsymbol{E}^{1}(x, t)\right|-\left|\boldsymbol{E}^{2}(x, t)\right|\right) \mathrm{d} x \geq \int_{\Omega} \boldsymbol{u}(t) \cdot \boldsymbol{e}(t) \mathrm{d} x \quad \text { for a.e. } t \in(0, T) .
\end{aligned}
$$

Adding (5.27) and (5.28) together implies

$$
\begin{aligned}
\int_{\Omega}- & \epsilon \partial_{t} \boldsymbol{e}(t) \cdot \boldsymbol{e}(t)-\mu \partial_{t} \boldsymbol{h}(t) \cdot \boldsymbol{h}(t) \mathrm{d} x \\
& +\int_{\Omega}\left(j_{c}\left(x, \theta(x, t), \boldsymbol{H}^{1}(x, t)\right)-j_{c}\left(x, \theta(x, t), \boldsymbol{H}^{2}(x, t)\right)\right)\left(\left|\boldsymbol{E}^{2}(x, t)\right|-\left|\boldsymbol{E}^{1}(x, t)\right|\right) \mathrm{d} x \geq 0 \quad \text { for a.e. } t \in(0, T) .
\end{aligned}
$$

As a consequence,

$$
\begin{array}{r}
\frac{1}{2} \frac{\mathrm{d}}{\mathrm{d} t}\|\boldsymbol{e}(t)\|_{\boldsymbol{L}_{\epsilon}^{2}(\Omega)}^{2}+\frac{1}{2} \frac{\mathrm{d}}{\mathrm{d} t}\|\boldsymbol{h}(t)\|_{\boldsymbol{L}_{\mu}^{2}(\Omega)}^{2} \leq \int_{\Omega}\left|j_{c}\left(x, \theta(x, t), \boldsymbol{H}^{1}(x, t)\right)-j_{c}\left(x, \theta(x, t), \boldsymbol{H}^{2}(x, t)\right) \| \boldsymbol{e}(x, t)\right| \mathrm{d} x \\
\text { for a.e. } t \in(0, T) .
\end{array}
$$

On the other hand, the Lipschitz property (A4) yields that

$$
\begin{aligned}
\int_{\Omega} \mid j_{c}\left(x, \theta(x, t), \boldsymbol{H}^{1}(x, t)\right) & -j_{c}\left(x, \theta(x, t), \boldsymbol{H}^{2}(x, t)\right)\left\|\boldsymbol{e}(x, t)\left|\mathrm{d} x \leq L\left(\|\theta\|_{\mathcal{C}\left([0, T], L^{\infty}(\Omega)\right)}\right) \int_{\Omega}\right| \boldsymbol{h}(x, t)\right\| \boldsymbol{e}(x, t) \mid \mathrm{d} x \\
& \leq \frac{L\left(\|\theta\|_{\mathcal{C}\left([0, T], L^{\infty}(\Omega)\right)}\right)}{2}\left(\underline{\epsilon}^{-1}\|\boldsymbol{e}(t)\|_{\boldsymbol{L}_{\epsilon}^{2}(\Omega)}^{2}+\underline{\mu}^{-1}\|\boldsymbol{h}(t)\|_{\boldsymbol{L}_{\mu}^{2}(\Omega)}^{2}\right) \quad \text { for a.e. } t \in(0, T) .
\end{aligned}
$$

Applying (5.30) to (5.29) results in

$$
\frac{L\left(\|\theta\|_{\mathcal{C}\left([0, T], L^{\infty}(\Omega)\right)}\right)}{2}\left(\underline{\epsilon}^{-1}\|\boldsymbol{e}(t)\|_{\boldsymbol{L}_{\epsilon}^{2}(\Omega)}^{2}+\underline{\mu}^{-1}\|\boldsymbol{h}(t)\|_{\boldsymbol{L}_{\mu}^{2}(\Omega)}^{2}\right) \geq \frac{1}{2} \frac{\mathrm{d}}{\mathrm{d} t}\|\boldsymbol{e}(t)\|_{\boldsymbol{L}_{\epsilon}^{2}(\Omega)}^{2}+\frac{1}{2} \frac{\mathrm{d}}{\mathrm{d} t}\|\boldsymbol{h}(t)\|_{\boldsymbol{L}_{\mu}^{2}(\Omega)} \text { for a.e. } t \in(0, T) .
$$

In view of this inequality and $\boldsymbol{e}(0)=\boldsymbol{h}(0)=0$, it follows that $\boldsymbol{e} \equiv \boldsymbol{h} \equiv 0$, and so (QVI) admits at most only one solution.

Existence. According to Theorem 5.1, the PDE-system (1.1) admits a unique solution $(\boldsymbol{E}, \boldsymbol{H}, \boldsymbol{J}) \in$ $W^{1, \infty}\left((0, T), \boldsymbol{L}^{2}(\Omega)\right) \cap L^{\infty}\left((0, T), \boldsymbol{H}_{0}(\mathbf{c u r l})\right) \times W^{1, \infty}\left((0, T), \boldsymbol{L}^{2}(\Omega)\right) \cap L^{\infty}((0, T), \boldsymbol{X}(\Omega)) \times L^{\infty}\left((0, T), \boldsymbol{L}^{\infty}(\Omega)\right)$. Thus, since we have proved that (QVI) admits at most only one solution, the assertion is valid, once we can prove that the solution $(\boldsymbol{E}, \boldsymbol{H})$ of (1.1) satisfies (QVI).

Multiplying the first equality in (1.1) by $\boldsymbol{v}-\boldsymbol{E}(t)$, with $\boldsymbol{v} \in \boldsymbol{L}^{2}(\Omega)$, and the second equality in (1.1) by $\boldsymbol{w}-\boldsymbol{H}(t)$, with $\boldsymbol{w} \in \boldsymbol{L}^{2}(\Omega)$, and then integrating the resulting equalities over $\Omega$, we obtain

$$
\begin{array}{r}
\int_{\Omega} \epsilon \partial_{t} \boldsymbol{E}(t) \cdot(\boldsymbol{v}-\boldsymbol{E}(t))-\int_{\Omega} \operatorname{curl} \boldsymbol{H}(t) \cdot(\boldsymbol{v}-\boldsymbol{E}(t)) \mathrm{d} x+\int_{\Omega} \boldsymbol{J}(t) \cdot(\boldsymbol{v}-\boldsymbol{E}(t)) \mathrm{d} x=\int_{\Omega} \boldsymbol{u}(t) \cdot(\boldsymbol{v}-\boldsymbol{E}(t)) \mathrm{d} x \\
\int_{\Omega} \mu \partial_{t} \boldsymbol{H}(t) \cdot(\boldsymbol{w}-\boldsymbol{H}(t)) \mathrm{d} x+\int_{\Omega} \operatorname{curl} \boldsymbol{E}(t) \cdot(\boldsymbol{w}-\boldsymbol{H}(t)) \mathrm{d} x=0 \quad \text { for a.e. } t \in(0, T) .
\end{array}
$$

Then, adding them together, it follows that

$$
\begin{aligned}
& \int_{\Omega} \epsilon \partial_{t} \boldsymbol{E}(t) \cdot(\boldsymbol{v}-\boldsymbol{E}(t))+\mu \partial_{t} \boldsymbol{H}(t) \cdot(\boldsymbol{w}-\boldsymbol{H}(t)) \mathrm{d} x+\int_{\Omega} \operatorname{curl} \boldsymbol{E}(t) \cdot \boldsymbol{w}-\operatorname{curl} \boldsymbol{H}(t) \cdot \boldsymbol{v} \mathrm{d} x \\
& \quad+\int_{\Omega} \boldsymbol{J}(t) \cdot(\boldsymbol{v}-\boldsymbol{E}(t)) \mathrm{d} x=\int_{\Omega} \boldsymbol{u}(t) \cdot(\boldsymbol{v}-\boldsymbol{E}(t)) \mathrm{d} x \quad \text { for a.e. } t \in(0, T) \text { and all }(\boldsymbol{v}, \boldsymbol{w}) \in \boldsymbol{L}^{2}(\Omega) \times \boldsymbol{L}^{2}(\Omega) .
\end{aligned}
$$


On the other hand, according to (1.1), $\boldsymbol{J}$ and $\boldsymbol{E}$ satisfy the Bean's critical-state law

$$
\begin{array}{ll}
|\boldsymbol{J}(x, t)| \leq j_{c}(x, \theta(x, t), \boldsymbol{H}(x, t)) & \text { for a.e. }(x, t) \in \Omega \times(0, T) \\
\boldsymbol{J}(x, t) \cdot \boldsymbol{E}(x, t)=j_{c}(x, \theta(x, t), \boldsymbol{H}(x, t))|\boldsymbol{E}(x, t)| & \text { for a.e. }(x, t) \in \Omega \times(0, T) .
\end{array}
$$

Consequently, it holds for all $\boldsymbol{v} \in \boldsymbol{L}^{2}(\Omega)$ and almost all $t \in(0, T)$ that

$$
\begin{aligned}
\int_{\Omega} \boldsymbol{J}(t) \cdot(\boldsymbol{v}-\boldsymbol{E}(t)) \mathrm{d} x & =\int_{\Omega} \boldsymbol{J}(x, t) \cdot \boldsymbol{v}(x) \mathrm{d} x-\int_{\Omega} j_{c}(x, \theta(x, t), \boldsymbol{H}(x, t))|\boldsymbol{E}(x, t)| \mathrm{d} x \\
& \leq \int_{\Omega} j_{c}(x, \theta(x, t), \boldsymbol{H}(x, t))|\boldsymbol{v}(x)| \mathrm{d} x-\int_{\Omega} j_{c}(x, \theta(x, t), \boldsymbol{H}(x, t))|\boldsymbol{E}(x, t)| \mathrm{d} x .
\end{aligned}
$$

Applying this inequality to (5.31), we conclude that $(\boldsymbol{E}, \boldsymbol{H})$ satisfies (QVI).

Remark 5.3. (i) As pointed out in the introduction, the eddy current approximation of the Bean critical-state model leads to a parabolic quasi-variational inequality [3]. While the existence for the corresponding twodimensional case is guaranteed, no uniqueness result was derived in [3]. In the case of the original Maxwell formulation (QVI), the uniqueness is satisfied thanks to the Lipschitz property (A4).

(ii) Our result extends $[27,30]$ due to the following reasons: First, we allow for simultaneous temperature and magnetic field dependence in the critical current. Second, our result holds true for all right-hand side $\boldsymbol{u} \in H^{1}\left((0, T), \boldsymbol{L}^{2}(\Omega)\right)$ and initial data $\left(\boldsymbol{E}_{0}, \boldsymbol{H}_{0}\right) \in \boldsymbol{H}_{0}(\mathbf{c u r l}) \times \boldsymbol{X}(\Omega)$ without any compatibility condition.

\section{FURTHER DISCUSSIONS}

The achieved well-posedness results open a way to analyze the temperature and voltage control in the magnetization process of type-II superconductivity. This leads to a state-constrained optimal control problem governed by (QVI). This problem requires a substantial extension of the developed results $[17,18,24,25,29,31]$ along with the recent results on the optimal control of QVI [1].

Acknowledgements. This work was supported by the German Research Foundation (DFG grants YO159/2-2 and YO159/4-1).

\section{REFERENCES}

[1] A. Alphonse, M. Hintermüller and C.N. Rautenberg, Stability of the solution set of quasi-variational inequalities and optimal control. SIAM J. Control Optim. 58 (2020) 3508-3532.

[2] J. Aponte, H.C. Abache, A. Sa-Neto and M. Octavio, Temperature dependence of the critical current in high- $T_{c}$ superconductors. Phys. Rev. B 39 (1989) 2233-2237.

[3] J.W. Barrett and L. Prigozhin, A quasi-variational inequality problem in superconductivity. Math. Models Methods Appl. Sci. 20 (2010) 679-706.

[4] J.W. Barrett and L. Prigozhin, Sandpiles and superconductors: nonconforming linear finite element approximations for mixed formulations of quasi-variational inequalities. IMA J. Numer. Anal. 35 (2015) 1-38.

[5] C.P. Bean, Magnetization of hard superconductors. Phys. Rev. Lett. 8 (1962) 250-253.

[6] C.P. Bean, Magnetization of high-field superconductors. Rev. Mod. Phys. 36 (1964) 31-39.

[7] M. Ciszek, B.A. Glowacki, S.P. Ashworth, A.M. Campbell, W.Y. Liang, R. Flükiger and R.E. Gladyshevskii, AC losses and critical currents in Ag/(Tl,Pb,Bi)-1223 tape. Phys. C: Supercond. Appl. 260 (1996) 93-102.

[8] J.R. Clem, B. Bumble, S.I. Raider, W.J. Gallagher and Y.C. Shih, Ambegaokar-Baratoff-Ginzburg-Landau crossover effects on the critical current density of granular superconductors. Phys. Rev. B 35 (1987) 6637-6642.

[9] G. Deutscher and K.A. Müller, Origin of superconductive glassy state and extrinsic critical currents in high- $t_{c}$ oxides. Phys. Rev. Lett. 59 (1987) 1745-1747.

[10] K.-J. Engel and R. Nagel, One-parameter semigroups for linear evolution equations, with contributions by S. Brendle, M. Campiti, T. Hahn, G. Metafune, G. Nickel, D. Pallara, C. Perazzoli, A. Rhandi, S. Romanelli and R. Schnaubelt. In: Vol. 194 of Graduate Texts in Mathematics. Springer-Verlag, New York (2000).

[11] V. Girault and P. Raviart, Finite Element Methods for Navier-Stokes Equations. Springer-Verlag, Berlin (1986).

[12] F. Jochmann, Well-posedness for Bean's critical state model with displacement current. J. Math. Anal. Appl. 362 (2010) $505-513$. 
[13] Y.B. Kim, C.F. Hempstead and A.R. Strnad, Magnetization and critical supercurrents. Phys. Rev. 129 (1963) $528-535$.

[14] A. Laurain, M. Winckler and I. Yousept, Shape optimization for superconductors governed by H(curl)-elliptic variational inequalities. SIAM J. Control Optim. 59 (2021) 2247-2272.

[15] J.-L. Lions and G. Stampacchia, Variational inequalities. Comm. Pure Appl. Math. 20 (1967) $493-519$.

[16] F. Miranda, J.-F. Rodrigues and L. Santos, Evolutionary quasi-variational and variational inequalities with constraints on the derivatives. Adv. Nonlinear Anal. 9 (2020) 250-277.

[17] S. Nicaise and F. Tröltzsch, A coupled Maxwell integrodifferential model for magnetization processes. Math. Nachr. 287 (2014) $432-452$.

[18] S. Nicaise and F. Tröltzsch, Optimal control of some quasilinear Maxwell equations of parabolic type. Discrete Contin. Dyn. Syst. Ser. S 10 (2017) 1375-1391.

[19] R. Picard, An elementary proof for a compact imbedding result in generalized electromagnetic theory. Math. Z. 187 (1984) 151-164.

[20] L. Prigozhin, On the Bean critical-state model in superconductivity. Eur. J. Appl. Math. 7 (1996) 237-247.

[21] J.F. Rodrigues and L. Santos, A parabolic quasi-variational inequality arising in a superconductivity model. Ann. Scuola Norm. Sup. Pisa Cl. Sci. 29 (2000) 153-169.

[22] J.F. Rodrigues and L. Santos, Quasivariational solutions for first order quasilinear equations with gradient constraint. Arch. Ration. Mech. Anal. 205 (2012) 493-514.

[23] W. Rudin, Functional analysis, 2nd edition. International Series in Pure and Applied Mathematics. McGraw-Hill, Inc., New York (1991).

[24] F. Tröltzsch and A. Valli, Optimal control of low-frequency electromagnetic fields in multiply connected conductors. Optimization 65 (2016) 1651-1673.

[25] F. Tröltzsch and A. Valli, Optimal voltage control of non-stationary eddy current problems. Math. Control Relat. Fields 8 (2018) 35-56.

[26] N. Weck, Maxwell's boundary value problem on Riemannian manifolds with nonsmooth boundaries. J. Math. Anal. Appl. 46 (1974) 410-437.

[27] M. Winckler and I. Yousept, Fully discrete scheme for Bean's critical-state model with temperature effects in superconductivity. SIAM J. Numer. Anal. 57 (2019) 2685-2706.

[28] M. Winckler, I. Yousept and J. Zou, Adaptive edge element approximation for $\mathbf{H}(\mathbf{c u r l})$ elliptic variational inequalities of second kind. SIAM J. Numer. Anal. 58 (2020) 1941-1964.

[29] I. Yousept, Optimal control of quasilinear $\boldsymbol{H}$ (curl)-elliptic partial differential equations in magnetostatic field problems. SIAM J. Control Optim. 51 (2013) 3624-3651.

[30] I. Yousept, Hyperbolic Maxwell variational inequalities for Bean's critical-state model in Type-II superconductivity. SIAM J. Numer. Anal. 55 (2017) 2444-2464.

[31] I. Yousept, Optimal control of non-smooth hyperbolic evolution maxwell equations in type-II superconductivity. SIAM J. Control Optim. 55 (2017) 2305-2332.

[32] I. Yousept, Hyperbolic Maxwell variational inequalities of the second kind. ESAIM: COCV 26 (2020) 34.

[33] I. Yousept, Well-posedness theory for electromagnetic obstacle problems. J. Differ. Equ. 269 (2020) 8855-8881.

\section{Subscribe to Open (S2O) A fair and sustainable open access model}

This journal is currently published in open access with no charge for authors under a Subscribe-to-Open model (S2O). Open access is the free, immediate, online availability of research articles combined with the rights to use these articles fully in the digital environment.

$\mathrm{S} 2 \mathrm{O}$ is one of the transformative models that aim to move subscription journals to open access. Every year, as long as the minimum amount of subscriptions necessary to sustain the publication of the journal is attained, the content for the year is published in open access.

\section{Ask your library to support open access by subscribing to this S2O journal.}

Please help to maintain this journal in open access! Encourage your library to subscribe or verify its subscription by contacting subscribers@edpsciences.org

We are thankful to our subscribers and sponsors for making it possible to publish the journal in open access, free of charge for authors. More information and list of sponsors: https://www.edpsciences.org/en/maths-s2o-programme 